\title{
Virtual and Mixed Reality in Telerobotics: A Survey
}

\author{
Costas S. Tzafestas \\ National Technical University of Athens, School of Electrical and \\ Computer Engineering, Division of Signals Control and Robotics, \\ Zographou Campus 15773, Athens, Greece (Email:ktzaf@softlab.ntua.gr)
}

\section{Introduction}

Virtual Reality (VR) constitutes now a well-established term, which is employed basically to describe systems that enable an intuitive and natural interaction in real-time between a human and a computer animated 3D graphical environment. The media that are employed to support such a human-computer interaction should ideally involve all human senses (and more generally sensori-motor abilities), that is, not only vision (through the rendering of 3D graphics models) but also audition, as well as the sense of touch, through a haptic interaction with virtual objects (Burdea \& Coiffet, 94). The challenge is to develop computer simulation environments (simulating an existent or fictitious world) in such a way as to create to the human user an illusion of reality. VR thus constitutes a multidisciplinary field covering a variety of scientific and technological subjects, from 3D computer graphics and multimedia technologies, to the design and control of mechatronic human-computer

$\varepsilon$ interaction devices, human factors and modeling of sensori-motor human skills, as well as

¿ topics related to the field of human perception and psychophysics.

$\underset{0}{0}$ VR and its applications have flourished significantly particularly during the last decade, not

only through the development of new software and hardware tools, devices and

ㄷ methodologies of human-computer interaction, but also by the implementation of these

$\frac{\mathcal{O}}{\mathrm{O}}$ techniques into a constantly increasing number of new application paradigms and human-

$\stackrel{\Phi}{.}$ centered activities. Augmented and mixed realities constitute in fact one form of applied VR

techniques and refer to the processes of overlapping synthetic (virtual) model images onto

real world images, that is, to the combination (mixing) of virtual and real world. In other

(1) words, in an augmented reality (AR) system virtual models complement or modify in a way

స్ల్ reality (providing in a sense some additional information as needed) instead of completely

을 substituting (replacing) reality, as is the objective in VR systems. The goal here is to provide

$\widetilde{\pi}$ the user with the illusion of 'co-existence' of virtual and real world at the same time and

of space domain.

\& Augmented Reality, as a research and technology field, has attracted considerable interest

$\underset{\leftarrow}{0}$ during the last years with new domains of practical applications arising. Much effort is

focused on the new potential opened by the application of AR techniques, and concern

$\stackrel{\square}{0}$ enhancing the ability of the human user (human operator) to perceive aspects of a real

world by overlaying additional (visual or other type of) information, increasing the abilities 
of interacting within this world. The auxiliary information that can be conveyed through the visual, haptic, or other form of display of virtual models may facilitate the user to perceive in a more direct and intuitive way specific "hidden" or else "fuzzy" characteristics of a real world, which may be needed for the efficient execution of a certain task. Therefore, while in general VR promises to revolutionize the way human-computer interaction systems are conceived, AR techniques seem to lead to the creation of excellent tools making execution of complex and demanding tasks more easy and intuitive for the human.

Virtual, Augmented and Mixed Reality technologies are now recognized as constituting a challenging scientific and technological field that can provide breakthrough solutions to a wide spectrum of application domains, where intuitive and natural human/computer and human/machine interaction is needed. Telerobotics, involving a human operator to control a robot from a remote location usually via a computer interface and a computer network, is one of the fields that can directly benefit from the potential offered by VR and AR human/machine interfacing technologies. Application of VR in Telerobotics (VRT) and the related concept of telepresence, or tele-symbiosis (Vertut \& Coiffet, 1984), are ideas which have been around for more than twenty years, and have been used mainly for the telemanipulation/teleoperation of robotic mechanisms in hostile environments (such as in the nuclear industry, space, underwater, or for other difficult or dangerous service/intervention tasks, like bomb disposal, civil works etc.). In fact, all the approaches involving the integration of VR techniques in telerobotics, as will be explained further in this chapter, constitute basically: (a) a generalization of the concept of "predictive displays", coping with the problem of time delay and stability in teleoperation systems, and (b) an attempt to provide human operator assistance and achieve better transparency characteristics for the teleoperation system.

Nowadays, on the other hand, the rapid development of new broadly expanded networking technologies, such as those related to the Internet, and the numerous relevant applications, described by the general term of e-commerce/e-business, can give new potential to the use of VRT in novel application domains. In fact VRT and Internet technologies can mutually benefit from ideas developed in the respective fields. This merging of technological potential can lead to a generalization of the concept of telework, where remote control through the network of actual physical processes will be possible. One can even think, for instance, of supervising and actively controlling a whole manufacturing process without having to move from his home. A major research objective must be of course to enable and promote new potential applications that can derive from the merging of such technologies, so that wider categories of the population can finally take benefit of these technological advances.

At the rest of this chapter, we focus on analysing the theoretical foundations of this field and on describing the practical application domains that are involved, by presenting some characteristic case studies. Section 2 starts with a description of the basic principles related to virtual and augmented reality systems, and then presents an overview of applications related to the field of robotics. In Section 3 we describe the basic concepts that govern telerobotic systems and present a historical survey of the field. Section 4 , then, presents typical application scenarios of these technologies, related to the two main robotic systems categories, namely robot manipulators and mobile robotic vehicles, and highlights the link with the new VR-based field of haptics. Concluding remarks and future research directions are given in Section 5. 


\section{Virtual and Mixed Reality: General Description}

During the last ten to fifteen years, Virtual Reality (VR), as a theoretical and applied research field, has attracted the interest of the international scientific community, as well as of the public opinion through extensive use of the term by the information, communication and entertainment media. However, the latter often results in an "abusive" use of this term, which is probably due to the lack of a formal definition of the field. In the sequel, we attempt to describe the basic principles that govern the field of VR, as well as of the more recent domain of Augmented and Mixed Reality systems, and we give an overview of related applications.

\subsection{Definitions and Basic Principles}

In an attempt to define what is a VR system, in relation to what can be seen as a simple human-computer or human-machine interaction, we can say that VR refers to: (a) computer generated and animated, three-dimensional realistic visualization space, enabling (b) real-time and multimodal interaction involving multiple sensori-motor channels of the human user, aiming to achieve (c) a sense of immersion and (virtual) presence in this synthetic (simulated) environment. The common factor here is, thus, the stimulation of human perceptual experience to produce an impression of something that does not really occur, but which is perceived and believed (potentially invoking, at some extent, human imagination) as being physically present and existing as a real world. The three important dimensions characterizing VR systems, and differentiating them from typical computer simulation environments, are: interaction, immersion and imagination, all contributing to create a sense of virtual presence and realism (Burdea \& Coiffet, 94).

A Virtual Environment (VE) created via graphics is a communication medium having both physical and abstract components. The three basic constituents of a VE are the content, the geometry and the dynamics (Ellis, 1995). The content consists of objects and actors. The geometry is a description of the environmental field of action, and has dimensionality, metrics (rules establishing an ordering of the contents) and extent (range of possible values for the elements of the position vector). Dynamics is represented by the rules of interaction among the VE contents, describing their performance as they exchange information or energy. The components of a VE are useful for enhancing the interaction of the operators with their simulations. Virtualisation is defined to be the process by which an observer (viewer) interprets patterned sensory impressions to represent objects in environment other than that from which the impressions physically originate. Virtualisation can be applied to all senses: vision, audition, contact, shape and position (haptic sense).

The three complementary technologies used to create the illusion of immersion in a VE are:

- Sensors (e.g. head position tracker or hand shape sensors)

- Effectors (e.g. stereoscopic displays or headphones)

- Special purpose hardware and software (connecting the sensors and effectors in such a way as to create experiences encountered by people immersed in a physical environment)

A general diagram showing the structure of a VR-based system and the linkages of its components is shown in Fig. 1. The human operator can interact with a VE presented by means of head and body referenced displays, the success depending on the fidelity with which sensory information is presented to the user. The environment experienced by the user via a VE simulation is of course imaginary. On the contrary, when referring to a 
teleoperation interface, the human operator is provided with a perception of an environment that is real (e.g. image views of the remote physical-task environment). One can then immediately consider the use of VR environments as intermediate representations "interfacing" the human operator with the remote task environment, with the objective being to assist him in several ways to perform more efficiently the desired physical task. In such teleoperation interfaces, real and simulated data can be combined via digital processing to produce intermediate environments of real and simulated (synthetic) objects. The mixture of real and virtual entities within the same environment refers to augmented and mixed reality interfaces, a new field that has evolved as a special category of VR systems, as described in the following section.

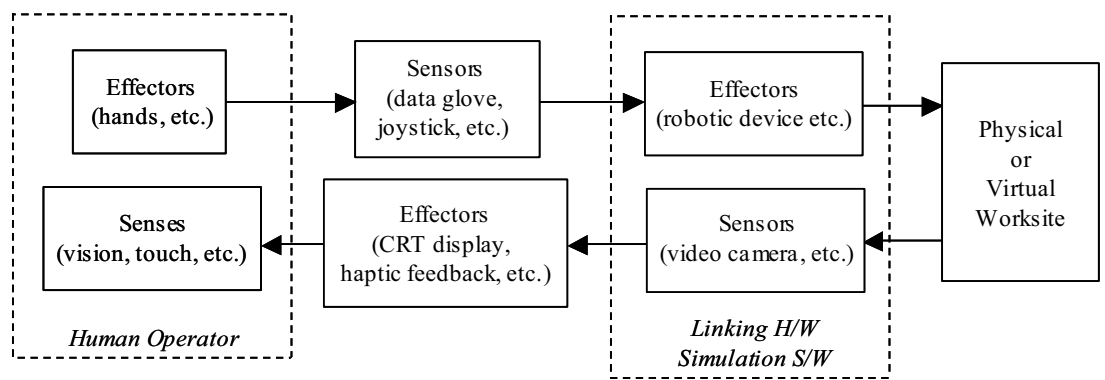

Fig. 1. General structure of VR-based human/machine systems.

\subsection{Augmented Reality: Basics}

Augmented Reality (AR) systems constitute in fact a category of quite specialized VR technologies, which have attracted significant interest during the last years due to the numerous applications they find in various new domains. While VR has as the main goal to immerse the human operator in a completely synthetic 3D simulation environment, the basic principle of AR systems is to enable the user to experience simultaneously parts of the actual (real) physical world. In other words, AR complements, instead of entirely substituting, the real world, with the ideal situation being to create the illusion that both virtual and real objects "coexist" in a unified (mixed) environment (Azuma, 97).

The question is then: why has this new field of AR systems found such an interest within the scientific community during the last years, and where does the usefulness of such mixed reality environments reside? The answer is that virtual environments can realistically display various data encoding complex information related to the real world, information that is not directly accessible in reality and could not be perceived differently from the human being. One can say that display of this information, which is conveyed by means of virtual objects, in fact "amplifies" the perceptual capacity of the human being, increasing the abilities to perform complex tasks on the real world.

The application domains of AR systems are various and are constantly evolving during the last years. They comprise: (a) medical applications, like simulation for education and training in medical (invasive or not) procedures, as well as pre-operative planning and computeraided (image-guided) operations, (b) CAD and manufacturing processes, for instance architectural design of a new building and "previewing" its spatial integration, training in maintenance procedures etc., (c) applications in the entertainment industry, e.g. with the production of special effects, virtual actors etc. Military applications are, unfortunately, also 
not excluded. In the following section, we present a short overview of VR and AR applications in robotics, as introduction to the use of such technologies in the field of telerobotics, which forms the main scope of this chapter.

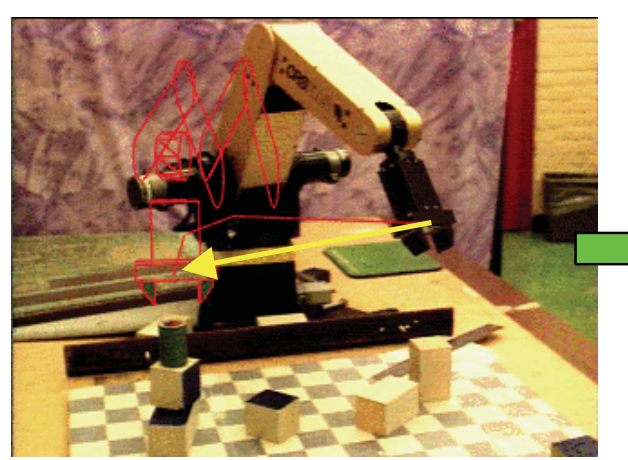

(a)

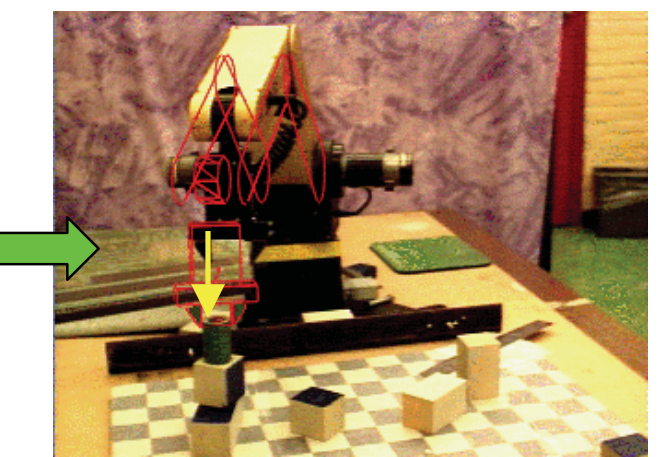

(b)

Fig. 2. Programming of a robot manipulator using augmented reality (AR) techniques. Overlaying virtual model ( $3 \mathrm{D}$ wireframe graphics above) on real robot image (adapted from: Rastogi et al., 96).

\subsection{Overview of VR Applications in Robotics}

A very important domain where $V R$ and $A R$ technologies find many and interesting applications concerns the field of robotics and robot integrated manufacturing systems. It is now asserted that the use of such technologies can provide significant benefits in all the phases of a manufacturing procedure, from initial design to implementation and control, particularly when these involve the integration of robotic systems. As outlined previously, given that a VE constitutes in fact an integrated human-machine interaction system, VR can contribute significantly in all the processes where human intervention (and human factor in general) plays an important role, like for instance:

- The design of virtual prototypes and the evaluation / assessment of various characteristics (including aesthetic, ergonomic etc.) and parameters related to functionality, feasibility tests, reliability, consistency of operation etc., which can be performed by means of interactive VR simulation environments.

- The programming and control of automated (robotic) procedures, with the goal being to better exploit the skills of the human operator, as well as his capacities to evaluate complex situations and solve decision problems, such as task and path planning involving a robot manipulator.

One very useful related application of AR concerns programming of robot manipulation tasks. The basic idea resides on the use of virtual models representing the robot and its task environment as an intermediate representation to guide and assist the robot action planning process. Overlaying 3D graphical models, representing the robot and the planned motion (action) sequence, on real views of the actual task environment, enables the human operator to perform a "previewing" of the system operation, potentially off-line, facilitating the programming and validation of complex robotic tasks, without the need to constantly work with the real robot in an on-line programming scheme (with all the advantages that such an 
off-line robot programming presents in practical scenarios). Of course, VR models must be correctly superposed on the real world images, for such systems to be of any practical use. This is called $3 D$ image registration, which constitutes one of the basic problems that needs to be tackled in any AR system, based on camera calibration techniques, and probably making use of (image- or sensor-based) 3D tracking methodologies (if such a system is to operate in real-time).

Fig. 2 presents an application example of such an AR-based programming of a robot manipulation task (Rastogi et al., 96). The graphical model (wireframe) shown in this figure constitutes in fact a virtual representation of the real robot, which is overlaid on real robot image views assisting the human operator to better evaluate the anticipated outcome of a programmed action sequence. In this case, the task consists of grasping an object and performing a "pick-and-place" operation. Fig. 2(a) shows the "predicted" path of the robot manipulator, as this is programmed using this AR interface. Fig. 2(b) displays a subsequent view with the robot manipulator having moved according to the planned operation, with both real and virtual robot images being registered (i.e. correctly aligned), demonstrating the accuracy of this robot planning scheme. It must be pointed out here that the additional use of stereoscopic images with 3D graphical models can significantly enhance the efficiency of the human operator in performing such robot programming tasks, by providing visual feedback information in a more intuitive way and increasing the overall performance of the system (reducing time, minimizing false maneuvers etc.).

Based on the above concepts, virtual and augmented reality techniques have evolved substantially during the last decade finding significant and interesting new applications particularly in a special field of robotics called telerobotics, which can be mainly characterized by the direct involvement of a human operator in the control loop. The theoretical and practical foundations of this domain form the main scope of this chapter and are thoroughly analysed in the following section.

\section{Telerobotics: Historical Evolution}

Telemanipulation as a scientific term describes all the methodologies and techniques enabling a human operator to perform from a distance a manipulative task, using his own hand through the use of an intermediate mechatronic system. Telemanipulation control of a remote manipulative task, besides its fascinating character related to the notion of extending human capabilities by some tool beyond usual space or time limits, it can prove extremely beneficial in cases where human intervention is indispensable to perform a task taking place in an unstructured "hostile" environment, due to the increased uncertainty and non-repetitiveness characteristics of such tasks, and the complex task/path planning required for timely and correct execution. Original master-slave telemanipulation systems consisted of a couple of mechanical or electromechanical arms (one called the master, controlled by the human operator, and the other, called the slave, performing the remote manipulation task). Bilateral exchange of energy (position and force signals) was initially ensured through a mechanical linkage and, later-on, through the use of electrical links and servo-control loops. In its infancy, telemanipulation technology found outstanding applications in the nuclear industry for the remote manipulation of radioactive materials in environments where human presence was hazardous. Typical example is the work accomplished by Raymond Goertz at Argonne National Laboratories, USA, or by Jean Vertut and the French group at the CEA (Vertut \& Coiffet, 84). 
Bilateral servo-controlled telemanipulation and industrial computer-controlled robotics were two technological fields developed originally in parallel and, in some extent, independently. The awareness that both these fields can benefit from development accomplished in each other has led to the fusion of these technologies and the creation of what is generally described under the term of telerobotics. Robotics was initially concerned with the development of industrial manufacturing systems performing programmable, repetitive operations in an autonomous sensor-based manner, while telemanipulation was focusing on a different class of tasks, which should clearly rely on the predominant presence of a human operator in the control loop. Telerobotics, which globally describes the fusion of these general technological fields, is a very challenging and promising research field, which aims at exploiting in a full extent both human operator skills and machine intelligence capabilities within a human/robot interaction and cooperation context.

The integration of some mobility characteristics on a remote manipulation system, has extended the workspace and, generally, the functionality of these systems in terms of space and task limitations, and has led to the creation of new application domains covered under the more broad term of teleoperation. Such application domains include the development of mobile telemanipulator vehicles for space operations (e.g. Mars Rover etc.), with typical examples being the mobile robotic systems developed by NASA, for future Mars exploration missions ${ }^{1}$. Underwater remotely operated vehicles (ROVs) have also been developed, such as those described in (Gracanin \& Valavanis, 1999). All these systems belong to the general field of intervention and service robotics, which focuses on the development of integrated mobile robot platforms with embedded manipulation and sensing modules, operating under direct remote control or semi-autonomously under highlevel human supervision. Such systems aim mainly at substituting the human being in the execution of hazardous (e.g. handling of explosives), painful (e.g. lifting heavy weights, for instance civil works), or else boring every-day tasks (e.g. vacuum cleaning etc.). In section 4.2, we will present one example of such a mobile service robot. This general field also comprises systems that aim at assisting humans when performing delicate operations, requiring increased precision, which is the case of the research performed in the field of medical robotics, dexterous telemanipulation and telesurgery.

Let's describe now the main problems encountered in general teleoperation systems, as well as some existing solutions, methodological approaches and guidelines proposed in the literature, in order to situate the current state-of-the-art of research carried out in the field of telerobotics. The major problem and certainly the most cited one is the presence of time delays in the bilateral communication loop, which is mainly due to the distance separating the master from the slave site, but may also be due to the processing time required for coding and data transmission. Such delays may be constant (e.g. in the case of direct ISDN link), but may also be varying in an unpredictable manner due to the load of the network servers (which is the case of the Internet), causing additional difficulties in coping with the problem. For instance, time delay for transcontinental teleoperation when a satellite link is used may exceed 1 second, while when teleoperating a rover on the moon, round-trip time delay approaches 3 seconds. The human operator is in such cases obliged to apply a "move-and-wait" strategy, that is, to make small moves while waiting for the images (and in general, the sensory feedback) to be updated. As a

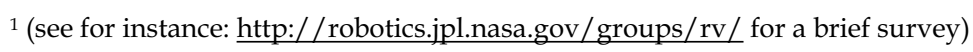


consequence, communication time delays cause certain degradation of the teleoperation system's performance; but what is even more critical, their presence may jeopardize safe operation and cause dangerous instabilities especially when force-feedback is involved in a long-distance bilateral telemanipulation system.

Degradation of sensory feedback may also be due not only to the presence of time delays and limited bandwidth, but also to noise and other sort of disturbances in the communication channel. Problems related to the quality of sensory feedback may also derive from the nature of the task itself, for instance when a slave robot operates in low visibility conditions (e.g. video feedback from an underwater remotely operated vehicle, which may, in some cases, be completely useless or extremely difficult to interpret). In all these cases, when sensory feedback is deteriorated, due to time-delays, noise or other source of signal degradation, some task-specific methodology or advanced remote control strategy has to be followed to assist the human operator to perform the task goals, and ensure safe and efficient operation of the system.

Time-delay has long been known in classical control theory as a very challenging problem, and various predictive control schemes have been proposed based on some a-priori knowledge of the delay (for instance, the predictor of Smith, proposed around 1956, see: (Laminat, 1993) for a survey). In the teleoperation field, more recently, some new control schemes have been proposed to cope with this problem, based on passivity theory (Anderson \& Spong, 1992), or on the concept of adaptive impedance (Niemeyer \& Slotine, 1991). All these approaches converge to the fact that, in any case, stability and transparency (defined in terms of force/trajectory tracking between the master and slave) of the teleoperation system are two contradictory objectives, and some trade-off between these characteristics has to be achieved most of the times. All these approaches in fact slow down the control system coupling the master with the slave, that is, diminish the control bandwidth of the system leading to a more compliant (less stiff) teleoperator. This ensures the stability (passivity) of the system, under some constraints related to the magnitude of the time delay, but has as a counter-effect to deteriorate the transparency of the teleoperation system (for instance, the human operator does not feel the real profile of the force generated at the slave site). The problem becomes even more difficult when time-delay is randomly varying, with no a-priori knowledge available on its order of magnitude.

Another class of techniques trying to cope with the problem of communication time-delay, is based on the use of predictive displays. Graphical predictors, supplying visual cues (estimations) on the evolution of the teleoperation task, are the most commonly used. Bejczy et al. (1990), for instance, have proposed the use of a wireframe graphical model of the slave robot, overlaid on the usual video feedback provided to the human operator. This combination of both synthetic and real images (that is the display of a graphical model, directly following the movements of the human operator and showing what the state of the robot will be before the actual delayed video images arrive from the slave site) greatly facilitates the task of the human operator. The paradigm of graphical predictive displays has been greatly adopted since, and extended to cope not only with problems related to the presence of time delays in the bilateral communication loop but also to perform visual feedback enhancement and assist the human operator in quickly assessing a situation and performing teleoperation tasks.

\subsection{Teleoperation and Virtual Reality: Synergy}

The integration of more advanced virtual reality techniques in teleoperation systems can be partly seen as a generalization of the concept of predictive displays described above, where the term display may now refer not only to the visual display of simple graphical cues, but 
also to other forms of sensory feedback such as haptic or auditive display. Virtual Reality is in fact a multidisciplinary scientific/technological field, which aims to enable a more natural and intuitive human/computer interaction based on the use of multimodal/multisensory interfaces. This human/machine interface technology involving various perceptuo-motor modalities of the human being (not only vision, but also haptic interaction and auditive feedback) can provide a technological solution of excellence for the human/robot interaction and communication systems constituting the field of telerobotics. Virtual environment simulations of teleoperation systems can indeed be used as predictive models performing the role of a mediator between the human operator and the remote (slave) robotic system. This means, in other words, that the human operator could be provided with realistic threedimensional graphical images of the remote operation site, while being able to interact with these images and perform the desired teleoperation task in a natural and intuitive way (that is, for instance, by feeling the reaction forces during the execution of this virtual task model), and all that before the actual (delayed or deteriorated) real sensory-feedback signals arrive from the remote slave site. In fact, this interaction between the human operator and the virtual environment (that is, the virtual task performed by the human operator) can be used to generate the appropriate command signals that have to be sent to the slave robotic site, and guide the on-line execution of the real teleoperation task. The use of such an intermediate virtual representation of a teleoperation task is reported in (Kheddar et al., 1997), where a multi-robot long-distance teleoperation experiment is described, as will be presented more in detail in Section 4.1.1.

VR-based models of teleoperation tasks can also be used in off-line teleprogramming schemes, in which case the master and slave control loops are completely decoupled. The human operator performs a virtual task in a completely simulated manner, within a 3D graphic environment representing the slave site. This virtual task is analyzed and the appropriate sequence of robot commands is extracted and recorded. The sequence of command signals is then evaluated by the human operator before its subsequent transmission to the slave robotic system, where real task execution will take place. Communication time delay is generally not a problem in this approach. However, this is not applicable for all kind of teleoperation tasks, for instance when fine telemanipulation of a dextrous robotic mechanism is required, since programming such complex tasks in the form of simple sensor-based operations is very difficult. The key issue in teleprogramming schemes is the type of commands that will constitute the robot programs, which must make use in full extent of any autonomy features supported by the slave robotic system, in the form of reactive sensor-based behaviours or elementary task operations. Such approaches are especially applied in super-long-distance teleoperation systems, for instance when guiding the operation of a rover on the surface of a distant planet such as Mars. Of course, the same idea of semi-autonomous teleoperation control can also be applied in an on-line direct teleoperation scheme, where more high-level command primitives can be send in real-time to the remote robot, instead of the traditional, continuous force/position/speed signals. In this general framework, Hirzinger et al. (1993) have proposed the use of a tele-sensor-based scheme for the remote control of a robot manipulator in space. Freund and Rossmann (1999) have proposed a task deduction/action planning approach (called projective virtual reality paradigm) tested on a variety of applications, from simple teleoperated robotic assembly tasks up to the control of multirobot telemanipulation systems for space applications. In 
Section 4.1.2, we will present an example of a similar telerobotic system, but with the application being that of a "remote laboratory" for education and training in robotics.

VR technology and its applications in different scientific fields have known a rapid development during the last five to ten years. We can now say with confidence that VR has the potential to become a key technology for the design of modern man-machine interfaces, as is the case of teleoperation systems. It can provide the tools and techniques to establish a multimodal, natural and intuitive human-machine interaction, increasing the feel of telepresence for the human operator, which constitutes the ultimate goal of any teleoperation/telerobotic system. Of course, many challenging problems have to be tackled and appropriate (generalized or task-specific) solutions must be proposed, taking into consideration not only ergonomic issues and human factors, but also more technical problems such as image calibration (Kim, 96), coping with discrepancies and modeling uncertainties, as well as control issues and stability of human-machine active interfaces.

The use of VR techniques in telerobotics can be seen as an evolution of general computeraided teleoperation schemes, developed to facilitate the task of the human operator and provide assistance in one of the following ways:

- by performing the functions of an information provider, that is, by enhancing the sensory feedback provided to the human operator and helping him to better understand the state of the remote task execution. Typical examples are the graphical predictive displays, described above, or some form of artificial haptic (kinesthetic and/or tactile) feedback. Other VR-based techniques include the use of virtual fixtures (Rosenberg, 1993) or virtual mechanisms (Joly \& Andriot, 1995).

- by performing some form of decision support function, that is, by providing suggestions or indications concerning the most suitable action plan and assist the human operator at the decision making process.

- by interpreting the actions of the human operator and performing a function of substitution or cooperation, to provide active assistance for the on-line control of a teleoperation task. This is the case of an active intervention of the master computer, with typical examples being a system undertaking the control of some degrees of freedom (dof), or ensuring that the commands issued by the human operator satisfy some constraints related to safety issues.

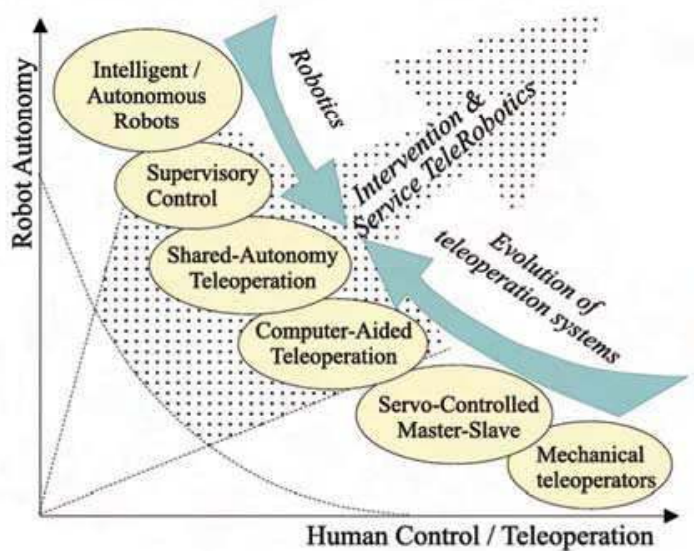

Fig. 3. Evolution of teleoperation systems towards intervention and service telerobotics. 
All these features (i.e. providing perception, decision or action assistance to the human operator) concern functions performed by the system within the master control station and are generally described by the term computer-aided teleoperation. Similarly, some form of computational intelligence can be embedded to the slave control system, which is for instance the case of a slave robot supporting some kind of autonomous sensor-based behaviors. In this case, we refer to a shared-control (or shared-autonomy control) mode of operation, with the slave robot executing a set of elementary (or more complex) operations in a completely autonomous mode. The commands issued by the master control station (that is, by the human operator) are described in a higher level of abstraction and include some form of implicit task representations. In an even higher level one could then think of a telerobotic system where the human operator is in charge of simply supervising the remote task execution, with active intervention only in extreme error recovery situations. All these paradigms are generally grouped under the term supervisory teleoperation, described in (Sheridan, 1992). A schematic representation of the evolution of these teleoperation paradigms is illustrated in Fig. 3. The interaction and merging of machine intelligence features with the human operator capacities and skills is the key issue that will lead to the creation of more advanced telerobotic systems, capable to perform more complex task such as those required in the field of intervention and service robotics. It is certainly one of the most challenging tasks for the designers of modern teleoperation systems, to find the "optimum line" between robot autonomy and human operator control, in order to exploit in a full extent the potential of such human/machine interaction and cooperation systems.

\subsection{Web-Based Telerobots}

Until quite recently, that is before the last five to ten years, telerobotic systems were remotely operated through dedicated fast network connections, and their use was exclusively reserved to trained specialists. The integration of teleoperation technology with new rapidly evolving media/network technologies, especially the Internet and the World Wide Web technologies, promises to open the door to a much wider audience, by creating and wide spreading new application domains. Controlling a real distant device over the Internet and performing a physical process in a remote location (as opposed to simple information processing) will extend the scope of telework applications, most probably having a significant impact in many aspects of both social and economic life. This section presents a brief survey of such web-based telerobotic systems. Situating the current state-ofthe-art for this promising and challenging research area, is of particularly interest within the scope of the survey presented in this chapter.

By web robots we mean robotic devices that are accessible from any computer connected on the Internet. Remote control of these systems via the Internet is possible by any site using a standard web browser incorporating the human operator control interface. Even though there exist by now many robots available for teleoperation on the web, the development of such systems is still more or less in its infancy and consists mainly of "playing" with a distant robot over the Internet, issuing simple motion commands to perform elementary tasks. A typical example is the Australia's telerobot, developed at the University of Western Australia ${ }^{2}$. It

\footnotetext{
${ }^{2}$ http:// telerobot.mech.uwa.edu.au/
} 
consists of a six-axis robot manipulator, remotely controlled with one fixed observing camera. The initial system, originally demonstrated in 1994, required users to type in spatial coordinates to specify relative arm movements. Since then, various user interfaces have been developed and tested (Taylor \& Dalton, 2000), which more recently embed Java technology to enable the human operator either to choose from a prespecified set of target positions or to click on the image and issue robot motion commands relative to the position of a cursor. The problem of course still remains to associate the position of the cursor that is being dragged on a 2D image, with the position of the robot end-effector and the other objects in the 3D world. An other very good example of a robotic manipulator being controlled over the Web is the PumaPaint system (Stein, 2000), which was on-line from June 1998 until March 2000. It consisted of a Puma 760 robot controlled over the Internet using a Java compatible web browser. The task performed by the robot was painting on an easel, reproducing in real the paintings created by the user on a virtual canvas, which was incorporated in the user interface running a Java applet. The interface forwards all commands to the robot so that almost the same image appears on the real canvas. The system also provides visual feedback in the form of periodically updated live images from the robot.

Besides these systems consisting of robot manipulators controlled through the Internet, there is another class of web robots involving teleoperation of mobile platforms over the www. Most of these systems provide exclusive remote control to a single person or provide queues to schedule user requests. One of the first mobile robots to operate in a populated office building, controlled through the web, was Xavier (Simmons, et al., 2000). This system was created by the end of 1995 to test the performance of various navigation algorithms, but has soon become very popular with more than 40,000 requests and 240 Kilometers travelled to date! The command interface of the robot provides a discrete list of destinations to send the robot and a list of simple tasks to perform there. When a user submits a task request, this task is scheduled for execution and a confirmation web page is sent back indicating when the robot will most likely carry out this task. If the user had registered using a correct e-mail address, the system will send an e-mail after completion of the requested task. In addition to the command interface page, there is a monitoring web page that includes the robot's current status, a map of the floor the robot is currently on and a picture of what it currently sees.

A very interesting application of such web-based systems involves remote control of mobile platforms moving in a museum. These are called tour-guide robots (Thrun et al., 1999), like the Rhino robot deployed in the Deutches Museum in Bonn, or its successor, Minerva (Schulz et al., 2000), installed successfully in the Smithsonian's National Museum of American History. These robots are operated either under exclusive control by remote users on the web (virtual visitors), or under shared control by both real (on-site) and remote (virtual) visitors of the museum. Under exclusive web control, the user interface is implemented as one Java applet incorporating a map of the exhibition area and two live images, one from the robot and the other from a ceilingmounted camera. Minerva's shared control interface was on-line for 91 hours and was accessed by 2885 people. The robot travelled $38.5 \mathrm{Km}$ under shared web and on-site control, providing information about 2390 exhibits.

There exist many other Web robots on the net, performing a variety of tasks such as those described in (Goldberg, 2000). The NASA Space Telerobotics program website ${ }^{3}$ currently

\footnotetext{
${ }^{3}$ http://ranier.oact.hq.nasa.gov/telerobotics_page/realrobots.html
} 
lists over 20 Real Robots on the Web. Reviewing all those web-based teleoperation systems, it is clear that the main problem is of course the unpredictable and variable time delay for communication over the Internet, which calls for the use of some form of supervisory control or off-line teleprogramming scheme to ensure stability. Most of the systems currently available on the web incorporate user interfaces, which implement basic functionalities, such as enabling the user to choose from a prespecified set of tasks (e.g. target locations). These interfaces use some combination of HTML forms or Java consoles to enter data and issue simple commands for immediate or future execution. Sensory feedback is usually limited to the display of images that are captured at the remote site, and the presentation of some status information in text form. It is obvious that this separation between the actions of the human operator (user) and the response of system fed back by the remote robot deteriorates the transparency and telepresence characteristics of the teleoperation system. More advanced "interactive telepresence" techniques need to be investigated, like for instance the integration of VR models and tools within the master control interface (including predictive displays and automatic active-assistance operations) to enable a more natural, intuitive and direct, real-time interaction between the user and the web-based teleoperation system.

\section{VR in Telerobotics: Application Scenarios}

As already stated, VR can be used in various ways to enhance robot teleoperation systems. Since it can be seen as constituting, in fact, a pool of advanced multimodal human/machine interaction technologies, VR can be employed at a "mediator" level between the human-operator and the remotely controlled robotic system. The performance of any telerobotic system can be measured in terms of two, often contradictory, indicators:

(a) Transparency, that is, the fidelity with which the human operator can perceive the remote robot environment, and the easiness by which he can perform the remote task via the telerobot, and

(b) Stability, particularly in the presence of large time delays in the bilateral communication and control loop that can jeopardize smoothness of operation, especially when force-reflecting bilateral telemanipulation is involved.

The goal of using VR interfaces as mediators in human-robot interactive communication systems would thus be twofold:

- to increase naturalness and intuitiveness of human operation, by: (i) enhancing information visualization via virtual and augmented reality displays, (ii) exploiting the use of multimodal sensori-motor interfaces taking into account human factors, and (iii) providing active assistance to the human operator; the goal of all these being, therefore, to improve transparency of the telerobotic system, facilitating the task from the human operator perspective.

- to cope with the presence of large time delays, (i) through the use of predictive displays by means of virtual and augmented reality models, and (ii) by applying some form of off-line teleprogramming scheme based on a virtual representation of the remote task environment; the goal being, here, to improve stability of operation and robustness for the telerobotic system. 

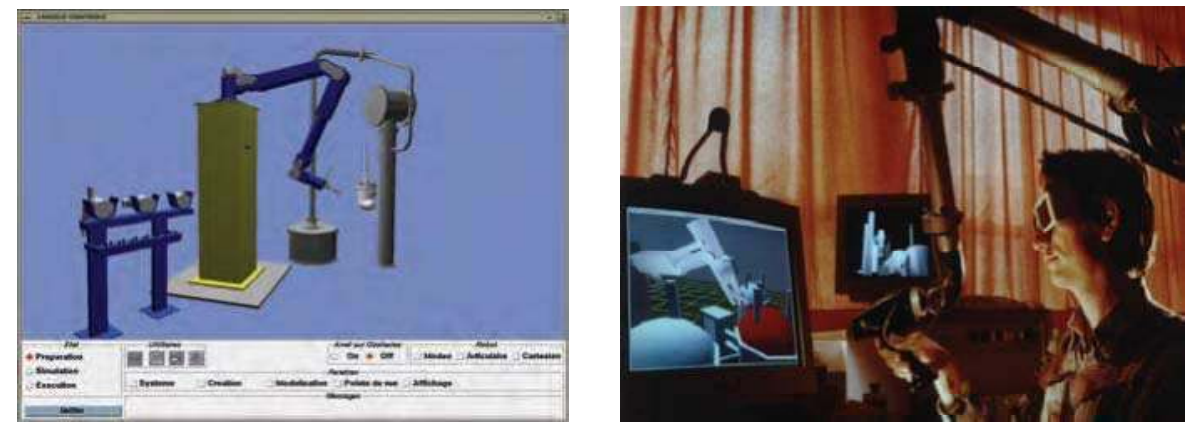

Fig. 4. TAO 2000 VR based teleorobotic interface (CEA, France).

In the rest of this section, we present some typical examples of VR-based teleoperation systems, for the two main classes of robotic systems, namely: (a) robot manipulators, and (b) mobile robotic platforms.

\subsection{Robot Telemanipulators}

Many experiments have been conducted, since over a decade now, in the context of robot telemanipulation, with main application fields being the nuclear industry (handling of radioactive material), and space telerobots (long-distance telemanipulation). The French Nuclear Centre (CEA Commissariat à l'Energie Atomique) is very active in the field, since the very beginning of the teleoperation history. One of the most recent advances is the TAO 2000 system, a VR based graphical programming interface, for nuclear servicing using a master-slave robot telemanipulator system. The whole system is illustrated in Fig. 4, with the graphical tele-programming interface (left) and the master force-feedback manipulator arm (MA-23, on the right). G. Hirzinger and his team (1993) at DLR 4 have developed a multisensory telerobot and conducted the first actual space experiments on a space telerobot technology (ROTEX). The system has flown in a space-shuttle mission and worked successfully in various modes, including autonomous operation, teleoperation by astronauts, as well as telerobotic ground control using either on-line direct teleoperation or, what was termed, a telesensor programming mode.

Since then, numerous telemanipulation systems have been developed, applying various methodologies adapted from the field of virtual and augmented reality, with many different applications (from telesurgery to nano-scale telemanipulation). In the sequel, we present two typical examples of VR-based robot telemanipulation systems based on the application of VR concepts and technologies: (a) a long distance, parallel teleoperation of multirobot systems, and (b) a distance training (remote / virtual laboratory) system, for teaching robot manipulator programming using a multimodal VR-based web-enabled interface.

\subsubsection{Long-Distance Multirobot Telemanipulation}

On October 10th 1996, a teleoperation experiment was performed involving four robot manipulators of different kinematics and situated in different locations (respectively Poitiers, Grenoble and Nantes in France, and Tsukuba in Japan). The robots were teleoperated simultaneously (in parallel) by the master control station situated in Poitiers.

${ }^{4}$ German Aerospace Research Establishment, Wessling 
This experiment was the first general one of a research cooperation programme named TWE (Telepresence World Experiment) linking seven research teams belonging to five countries (among them, the Laboratoire de Robotique de Paris in France, and the Mechanical Engineering Laboratory in Tsukuba, Japan).

The main challenge of this "telework experiment" was to demonstrate the possibility offered by VR technologies to ameliorate the human operator master control interface and enhance the capabilities of such robot teleoperation systems. With four different robots controlled in parallel to perform the same task, a common intermediate representation is imperative, to let the human operator focus on the task to be performed and 'mask' any robot manipulator kinematic dissimilarities and constraints. Fig. 5 shows an overview of the experimental setup, with the master control interfaces, and two robots in parallel operation (one in France and one in Japan). As can be seen in this figure, the task consisted of assembling a four-piece puzzle within a fence on a table. The operator performs the virtual puzzle assembly using his own hand and skill on the master control virtual environment, constituting an intermediate representation of the real remote assembly task. The visual and haptic feedback is local and concerns only the graphic representation of the remote task features without any remote robot. The operator / VE interaction parameters are sent to another workstation in order to derive robot actions (graphically represented for software validation and results visualization) and does not involve any direct operator action/perception. A video feedback was kept for safety and error recovery purposes.

The ultimate goal of such research efforts is to study the role that VR concepts and techniques can play towards the development of "efficient" interaction and communication interfaces between humans and robots. In the direction of ameliorating the transparency of the telerobot system, which constitutes a major target as has been already stated, such a human-robot interface must enable the human operator:

- to remotely perform the desired task in a natural and intuitive way, as if he was physically present at the remote (slave robot) site, without feeling obstructed or constrained by the telerobotic system, an objective that is often described by the term "telepresence",

- to use his manual dexterity in remotely conducting the desired manipulation task, meaning that the system must support natural skill transfer between the human operator and the remotely controlled (slave) robot.

To approach these key objectives, it is particularly important for the master teleoperation environment to display information to the human operator not in a static way but through a multimodal / multisensory dynamic interaction environment. VR concepts and tools play a significant role in this direction. Particularly, interacting via the (active) sense of touch is of primary important. This is termed "haptic interaction", or haptics, which is now a very active research field worldwide, with numerous applications, as we will see later on in this chapter. These haptic systems are often based on the development of special purpose glove-like devices, often termed "data-gloves", or "force-feedback gloves" if application of forces on the human-operator's hand is possible. One such example is illustrated in Fig. 6, where the human-operator wearing a specially designed exoskeleton device on his hand (the LRP dexterous hand master, see for instance (Tzafestas et al., 1997)) can interact, in a direct and intuitive way, with a virtual environment representing the task to be performed. The virtual manipulation actions performed within this VR simulation environment on the master control site are transformed into an appropriate sequence of commands for a robot manipulator to execute, and are then transmitted to the slave robot site(s). 


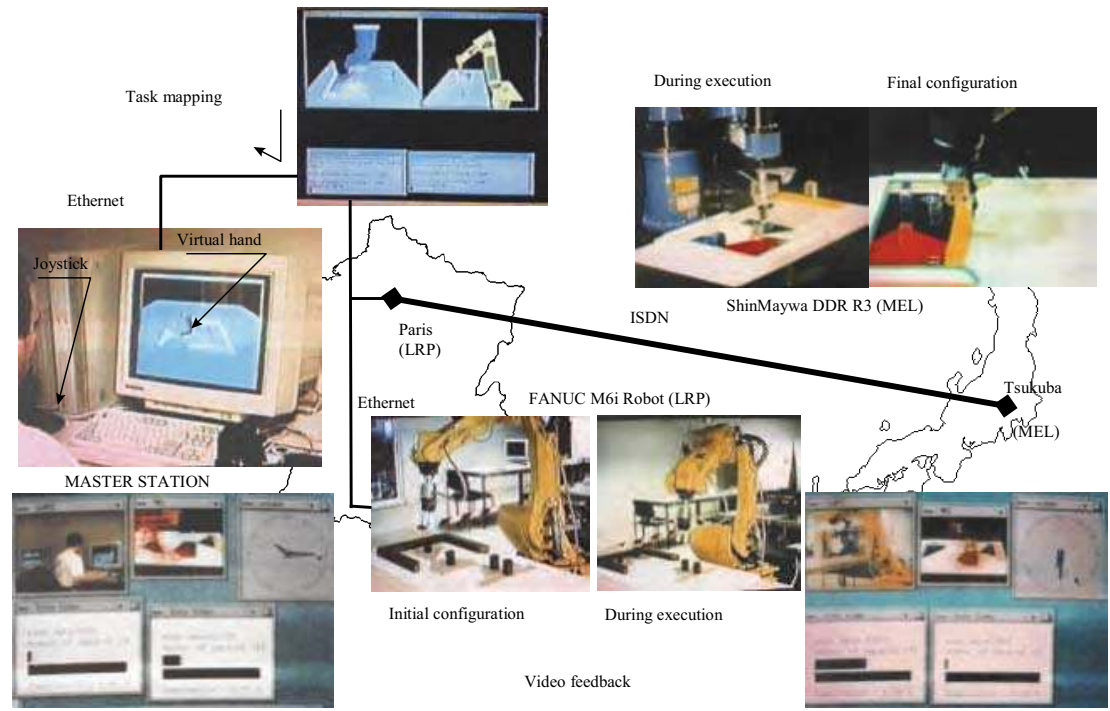

Fig. 5. Multi-robot long-distance teleoperation experiment (adapted from (Kheddar et al., 97)).

This general concept according to which, in an ideal telerobotic system, the human operator must feel as if he directly performs the task, instead of controlling the robot to perform the task, was called the "hidden robot" concept, and was also applied in the context of the multirobot teleoperation experiment depicted above in Fig. 5.

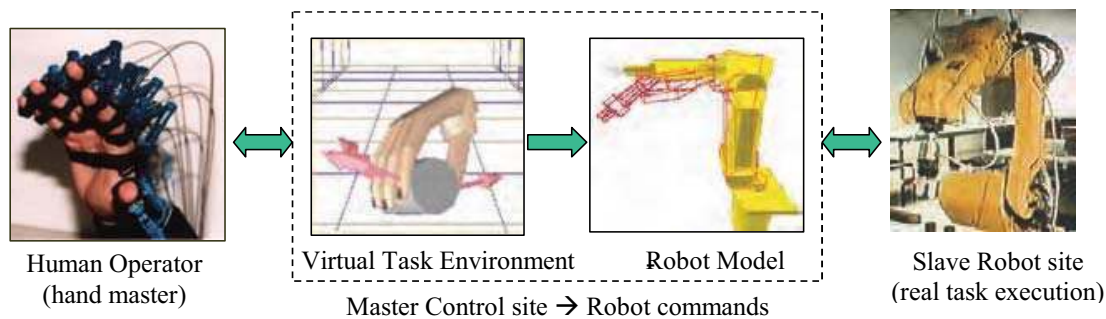

Fig. 6. Robot teleoperation by means of a virtual tele-work environment (adapted from (Kheddar et al., 97)).

The idea here is to let the human operator concentrate his awareness only on the task at hand and not on both the robot control and the task. The human operator is, thus, not concerned with the constraints imposed by the robot mechanisms (e.g. kinematical dissimilarities, etc.). Such issues are resolved by the system and are transparent to the user, giving him the opportunity to better concentrate on the task to accomplish. Of course, the system must possess adequate "intelligence" to interpret correctly the human manipulative actions performed within the master virtual environment. It must analyse these actions, extract the critical task parameters and deduce (in real-time, in case of direct teleoperation, or off-line, in case of teleprogramming) the commands that need to be sent to the slave robot for execution. 
We can thus conclude that the application of VR-based concepts and tools in the control of robotic telemanipulation systems, aims principally the development of a human-robot interactive communication framework that allows to exploit in a better extent: (a) from one hand, the dexterity and skills of the human operator to conduct dexterous manipulation tasks and solve complex decision problems, and (b) on the other hand, the capacities of robot manipulators to execute, with superior speed and accuracy, a combination of sensor-based primitive tasks.

\subsubsection{Distance Training in Robot Manipulator Programming}

Substantial application scenarios of VR technologies can be found in the field of education. If these technologies are combined with teleoperation concepts and tools they can lead to the development of very efficient remote and virtual laboratory platforms, aiming to enable distance training in a number of engineering disciplines. One such application is described in (Tzafestas et al., 2006), presenting a platform that aims to enable student training in robot manipulation and control technologies from any remote location via Internet. Access to robot manipulator arms and other similar mechatronic devices and laboratory equipment is often either limited by specific time restrictions or even not provided at all. One prohibitive factor is the high cost of such equipment, which makes it very difficult for many academic institutes to provide related laboratory training courses in their educational curricula for engineers. Therefore, the benefits from providing a means for any-time/any-place (virtual and/or remote) experimentation, in a "lab facilities sharing" context, are evident from a socio-economic point of view, apart from a pedagogical point of view related to the completeness and quality of practical training possibilities offered to their students.

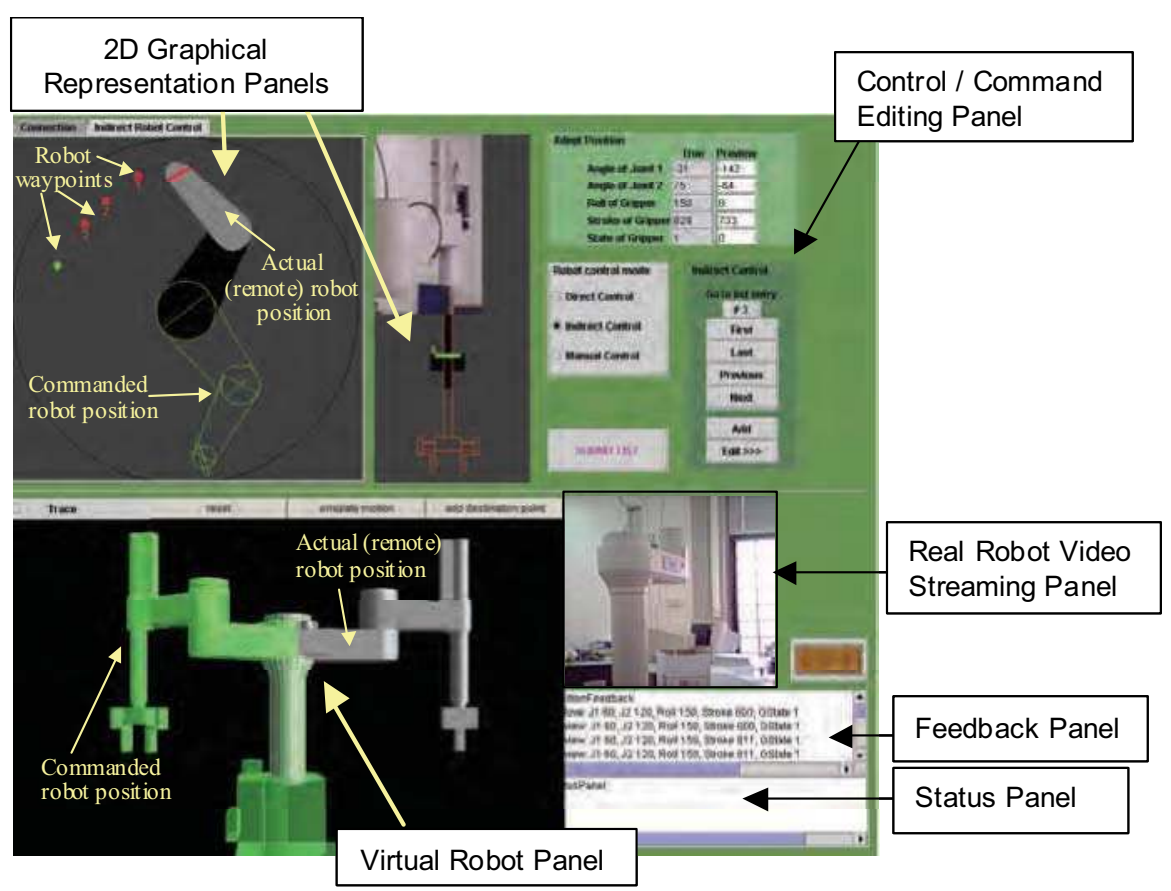

Fig. 7. The graphical user interface of the virtual robotic laboratory platform. 
Taking into account these considerations, the work described in (Tzafestas et al., 2006) was directed towards the development of a virtual robot laboratory platform that will train students on how to program a robot manipulator arm, using the functionality and programming modalities provided by the real robotic system. The platform developed incorporates a robot's Teach Pendant emulator, as well as a virtual 3D robot animation panel integrated in the graphical user interface. The system enables students to create, edit and execute robot programs (i.e. complete motion sequences, such as a pick-andplace task), in exactly the same way as they would if they were using the real-robot's pendant tool. The program created can be previewed "locally" by the student/trainee in 2D and 3D animation modes, and can then be sent for execution: either (a) by the virtual robot simulation, incorporated as mentioned above in the graphical user interface, or (b) by a real, remotely located, robot manipulator (such as, in this case, a SCARA-type AdeptOne manipulator located in the premises of the robotics laboratory).

Fig. 7 shows the graphical user interface (GUI) of the virtual and remote robotic laboratory platform, which is developed based on Java technologies and integrates the following control panels:

- 2D graphical representation panels (top-view and side view), visualizing both actual and commanded robot configurations,

- a real-time video streaming panel, which is based on RTP and implemented using JMF, showing (when on-line) the real remote manipulator in motion,

- a control/command editing panel,

- an interactive panel providing an exact emulation of the robot's Teach Pendant, called Virtual Pendant,

- status and feedback panels providing real-time textual information on current robot state, and

- a virtual robot panel, implemented using Java3D API, providing 3D visualization of both the commanded (preview animation) and the current robot configuration.

The overall architecture of the remote laboratory platform is depicted in Fig. 8. The system is based on a client-server architecture, enabling users to connect via Internet (or LAN). It supports multiple connected users through the implementation of a specific protocol using TCP/IP sockets for communication and real-time data exchange with the "robot server". The robot server supports the following three remote control modes: (i) direct teleoperation control, (ii) indirect control, for robot teleprogramming via the command/ editing panel, and (iii) manual control, that is, robot manipulator programming using the Virtual Pendant functionalities. These control modes are inspired from the telerobotics field, and particularly from work proposing various "shared-autonomy" and "supervisory" remote control modalities. In direct teleoperation, every command issued by the user (human operator) locally, i.e. within the GUI (master control site), is immediately transferred for execution to the remote (slave) robot. At the same time two types of feedback displays are active: (a) a predictive display (both in the 2D and 3D graphical panel) immediately visualising the commanded robot motion according to the human operator issued commands, and (b) a real robot feedback display (also both in 2D and 3D animation), showing where the robot actually is (that is, visualising current remote robot configuration, information provided in real-time through continuous feedback from the remote site). 


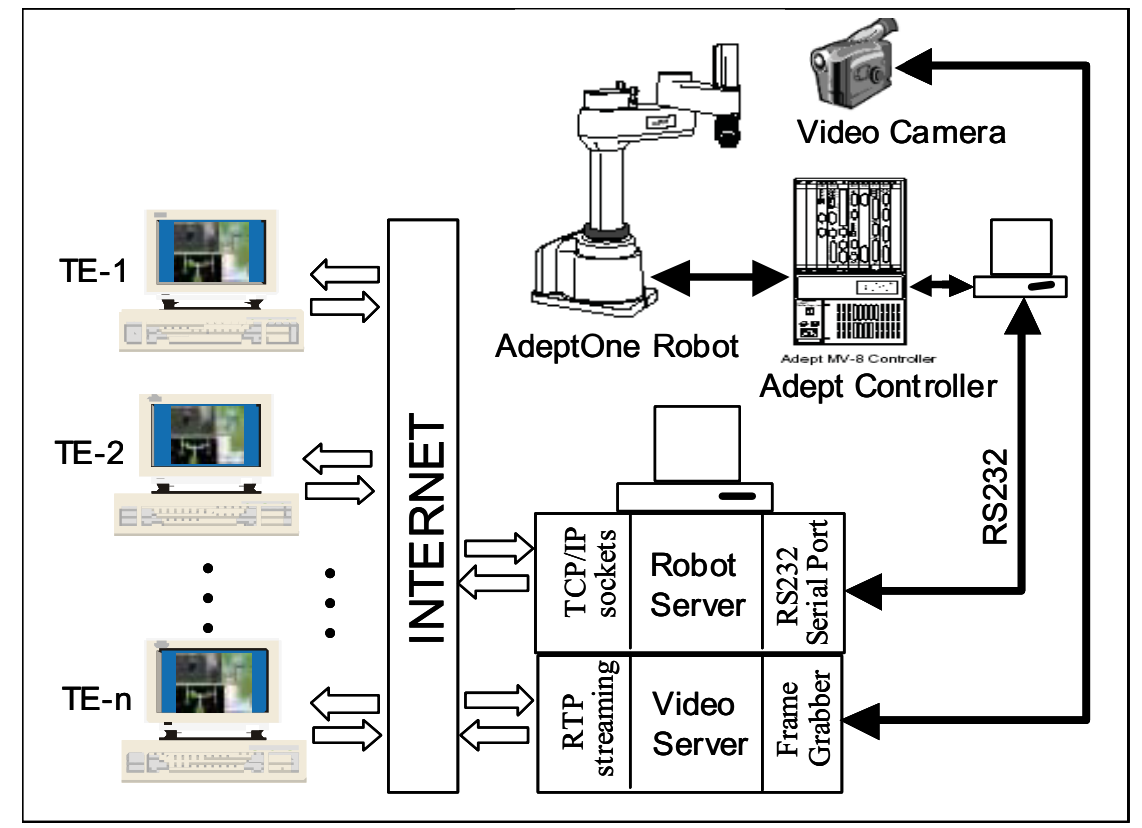

Fig. 8. Overall Architecture of the Virtual and Remote Robot Laboratory Platform.

As opposed to direct teleoperation, in the indirect "teleprogramming" control mode the commands are generated off-line, queued in a list and submitted to the robot in a subsequent time frame, when the human operator decides to do so. The idea is to be able to create a complete robot program off-line, test its validity and optimality, before actually sending the command sequences for execution by the real robot. Based on the functionality (robot command editing routines, waypoints list creation etc.) of this indirect teleprogramming mode, a third "manual-control" mode has been developed, which implements exactly the Virtual Pendant robot-programming scheme. This Virtual Pendant panel supports all the main functions of the real robot's pendant tool, and enables the student to learn and practice robot-programming routines locally.

A pilot study was conducted to evaluate the performance of the virtual and remote robotic laboratory platform. The objectives of this study were: (1) to explore to which extent the considered e-laboratory modalities can be efficiently implemented in practice and used by students to obtain practical training as a supplement to a theoretical course module (in this case, an introductory course on robotic manipulation), and (2) to explore the relative importance of various e-learning elements, particularly virtual vs. remote training modalities, in comparison with traditional hands-on experimentation. Experimental results show that students trained using the virtual training modality performed equally well as compared to students trained the classical way on the real robot, and were even seen to be more motivated, as revealed by the error rate related to the assimilation of high-level concepts and skills. These results are very interesting since they show that VR technologies and tools, when combined with telerobotic concepts and techniques in distance training scenarios, can prove very beneficial and contribute significantly to the development of very efficient virtual learning platforms, in many engineering (and not only) disciplines where 
laboratory / hands-on training is of primary importance to complete a full educational curriculum. Of course, larger scale studies are still needed to draw more general conclusions regarding the feasibility of these goals and the acceptability of such new technologies by students in their education and training practice.

\subsection{Mobile Robotics}

Control of mobile robots is often considered as being identified with the field of autonomous and intelligent robotics. From the very early work in this field (for instance, the mobile robot Shakey ${ }^{5}$ developed at Stanford University in the early 70's), mobile robots have been designed as machines that are supposed to work in autonomous fashion, according to variations of a straightforward "sense-think-plan-act" control architecture. Mobile robots must sense their environment, reason about it, plan their actions and move around autonomously. However, due to the complexity of such tasks and the limitations encountered as "bottlenecks" in the field of artificial intelligence, the presence of a human operator in the loop, being able to take control of the system, is considered imperative especially in unstructured and highly uncertain environments. The tendency is to design robotic systems that support some type of "intelligent human-robot cooperation", in line with the principles developed in the field of telemanipulation, and related schemes such as: shared autonomy control, supervisory control, teleprogramming, etc.

The application of virtual and augmented reality techniques for the teleoperation of mobile robots follows the same guidelines as in the case of telemanipulators, with similar objectives being: (a) to assist the human operator and facilitate remote control by means of a highly interactive and intuitive interface, (b) to enhance information feedback through augmented reality models, (c) to support off-line mission planning and teleprogramming of the mobile robot by means of a VR-based graphical user interface. Mobile robot platforms can use any type of locomotion, namely: wheeled (indoor/outdoor), legged (walking robots), airborne, underwater, space robots etc. Of course, depending on the robot's environment (e.g. visibility conditions, rough terrain, distance from master station, communication bandwidth, etc.) and on the locomotion mechanisms (e.g. fast vs. slow moving robot, stability during motion etc.), the teleoperation interface should adapt accordingly to cope with task specific problems.

A typical application domain, where advanced teleoperation technologies are used in the field of mobile robotics, concerns underwater robots (Remotely Operated Vehicles - ROVs). One such example is presented in (Sayers et al., 95), where a teleprogramming concept was applied in the frame of a subsea ROV equipped with a robotic arm. The main problems in such systems concern: (i) the limited communication bandwidth (of some Kbits/sec) resulting in a round trip communication delay up to 7 seconds, which is typical of the subsea acoustic transmission channel; (ii) the limited visibility and distorted images received at the master control station, typical of underwater operation; and (iii) the effect of underwater currents on the mobility of the ROV platform. The use of virtual and augmented reality models as predictive displays in the master control GUI can help alleviate some of the difficulties related with this specific application environment. Figure 9 shows snapshots of the system in operation, both from the operator's interface and from the real robot performing a grasping task. However, the most exceptional instance highlighting the use of

${ }^{5}$ See: http://www.ai.sri.com/shakey/ for more information 

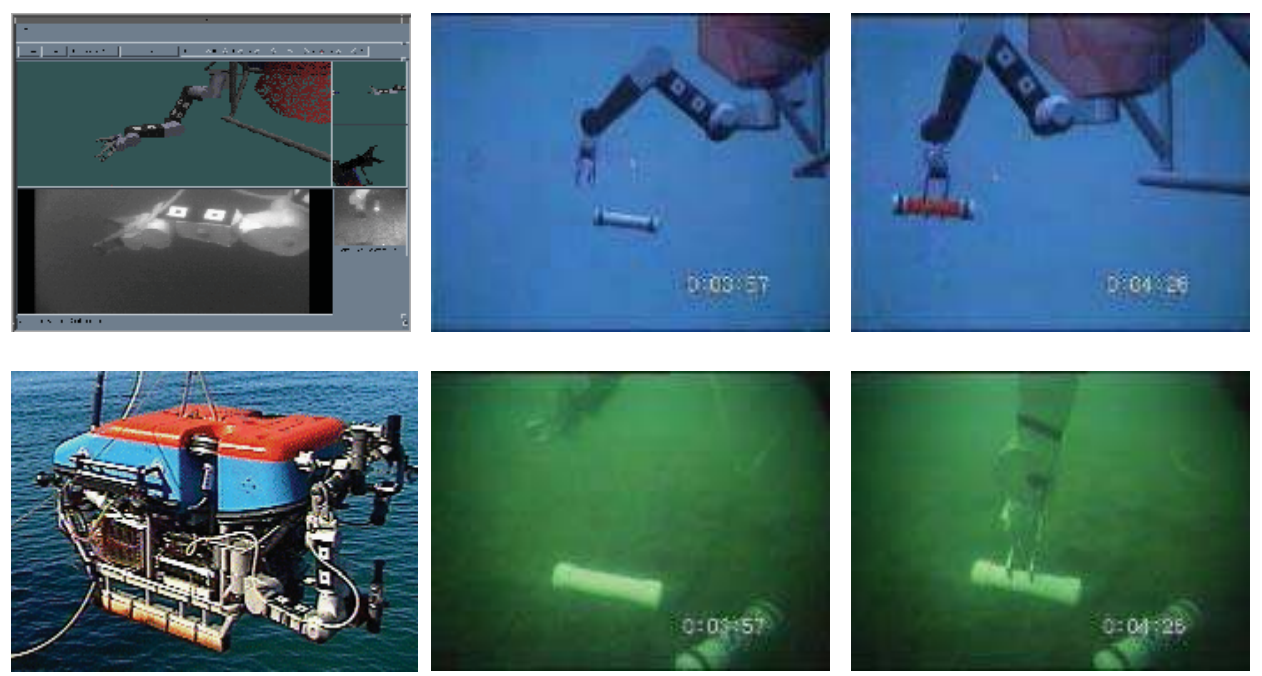

Fig. 9. Teleoperation of a subsea ROV. Up-line pictures designate the master control station, and down-line pictures show an image of the real submersible robot, as well as snapshots of the video feedback from the robot (JASON ROV) environment (adapted from (Sayers, 99), the GRASP laboratory, Pennsylvania University).

VR techniques in mobile robot teleoperation is undoubtfully the Mars Pathfinder Mission (NASA) ${ }^{6}$. Indeed, a VR technology based supervision and control workstation was designed to remotely command the Sojourner rover, which landed on the Mars planet by July 4th 1997. A good survey of these VR-based teleoperation technologies and their applications can be found in (Kheddar et al., 2000).

In the rest of this Section, we present two application examples regarding the use of VR techniques in mobile robot teleoperation. The first one concerns a mobile robotic assistant teleoperated in a modelled indoor navigation environment, while the second one focuses on the use of more advanced haptic interface to facilitate remote exploration of an unknown environment using a miniature mobile robot.

\subsubsection{Teleoperation of a Mobile Robotic Assistant}

The work presented in this paragraph was carried out in the framework of a research project called "HygioRobot" (Health-Robot), funded by the Greek General Secretariat for Research and Technology and the European Commission. The aim of the project was the development and implementation of control algorithms for a mobile service robot, consisting of an integrated robotic platform equipped with a vision system and a light manipulator (Tzafestas et al., 2000). The system was targeted towards a particular class of applications, namely to perform assistive tasks in a hospital environment.

${ }^{6}$ See: http://mars.jpl.nasa.gov/MPF/ and http:// www-robotics.jpl.nasa.gov/ for details 


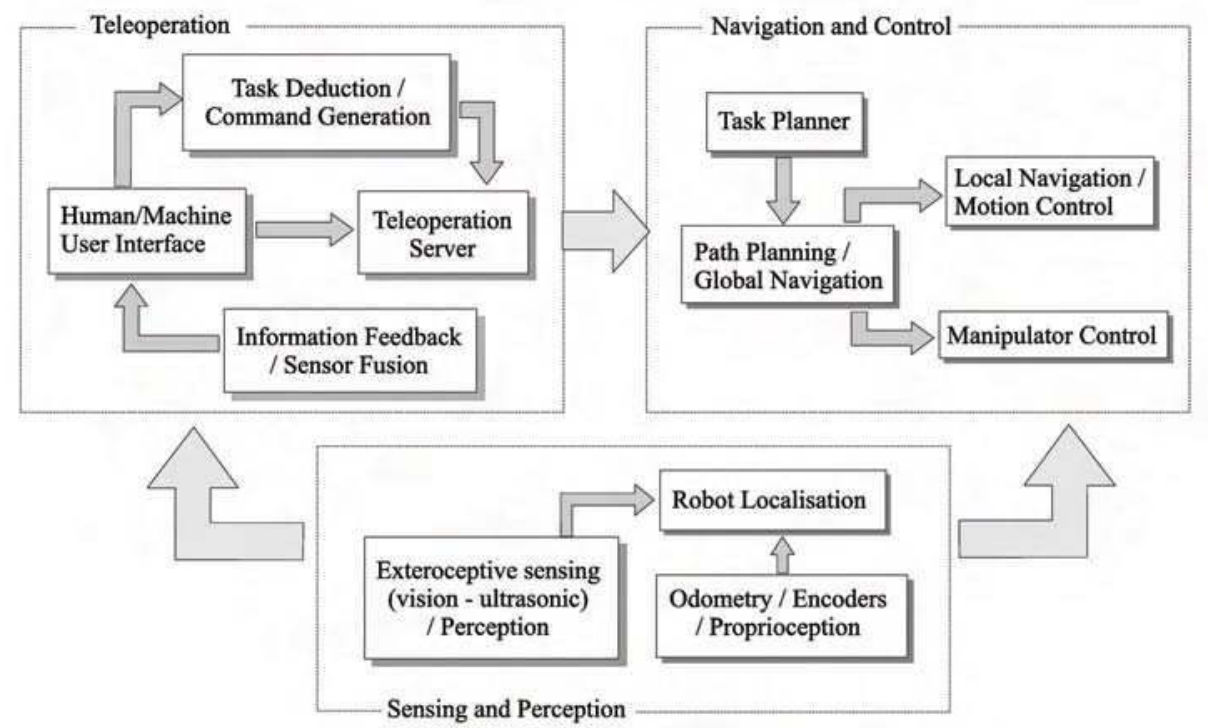

Fig. 10. Teleoperated mobile robotic assistant: general architecture of the system (adapted from (Tzafestas et al., 2000))

These may include tasks such as transportation of specific items (like pharmaceuticals, lab specimens, medical records etc.), accompanying a patient from one location to another within the hospital building, or even surveillance of an area, in other words, a combination of simple displacement and manipulation (fetch-and-carry) operations in a potentially human crowded indoor environment.

The global architecture of the system, with the principal functional modules and their interconnections is shown in Fig. 10. It consists of the following main subsystems:

(a) The navigation and control subsystem, including the task planner, the global and local path planning and navigation modules, as well as the manipulator control module. Major issues that must be investigated include here: (i) the real-time collision avoidance to ensure safe operation of the mobile platform in a dynamic environment, (ii) the development of a number of autonomous low-level sensor-based behaviors, and (iii) the coordinated action of the mobile platform and robot manipulator to optimally exploit the redundancies offered by such an integrated mobile manipulation system,

(b) The sensing and perception subsystem, performing fusion and interpretation from a variety of sensory feedback information, provided by the odometry and optical encoders (proprioceptive feedback), as well as from the vision and ultrasonic sensors (exteroceptive feedback). The goal of this subsystem on its whole is to update internal representations regarding: (i) the robot's actual state (positioning, i.e. robot localization) and (ii) the external world map (i.e. dynamic moving obstacles etc.)

(c) The teleoperation subsystem, which aims at integrating the decision and action capacities of a human operator in the control loop, and consists of: (i) a multimodal user interface (which will be designed to enable Web-based remote control of the 
robotic platform), (ii) a sensory feedback acquisition and processing module, and (iii) a task deduction and command generation subsystem. All these modules are coordinated by a teleoperation server, which was designed to support various modes of operation ranging from direct on-line remote monitoring and control, to off-line teleprogramming or simple supervisory control of the system.

The work described briefly in the sequel focuses more specifically on the design and implementation of a multimodal teleoperation system for the mobile robotic assistant, integrating virtual reality techniques within a Web-based user interface, to assist the human operator and enhance the functionality and efficiency of the system. Efficiency in remote operation and control of a robotic system concerns: (a) making "good use" of the available communication bandwidth between the master and slave systems, and (b) achieving a "synergy" between the human operator and the robot, by enabling the system to best exploit and integrate (in terms of speed, precision and error recovery) both (i) the human operator capacity to take rapid decisions and intuitively indicate the most appropriate (coarse or detailed) plan for system action (e.g. robot motion) in complex situations, and (ii) the robotic system capacity to perform, with controlled speed and precision, a variety of autonomous operations.

To approach towards these general targets, a set of requirements have to be specified and fulfilled by the teleoperation system and all its sub-modules. The final system design must converge towards the merging between a number of often contradictory modalities, in search of an "optimum" compromise and increased "efficiency". By multimodal teleoperation interface we mean a system that supports: (a) multiple computer-mediated human/robot interaction media, including VR models and tools, or even natural gesture recognition etc., and (b) multiple modes of operation, with a varying degree of robot autonomy and, respectively, human intervention. The latter is a very important issue for the design of a telerobotic system, as has been already cited in previous sections. The modes of operation that have been considered for this teleoperation system included:

(a) Direct teleoperation control, based on on-line exchange of low-level commands and raw sensory signals.

(b) Computer-aided teleoperation of the mobile robot, with the master control system providing some form of assistance to the human operator, such as: (i) performing information feedback enhancement, like model-based predictive display, (ii) undertaking active control for some of the degrees of freedom (dof) of the system, substituting or complementing the human operator, or even (iii) providing some form of active guidance and model-based correction for the human operator's actions. Two main functions have been integrated in the system: an active anti-collision and an active motion-guide function, both based on the use of either a virtual reality model of the robotic platform and its task environment, or of a simple 2D topview representation.

(c) Shared-autonomy teleoperation control of the robotic system, using a set of sensorbased autonomous behaviors of the robot. This mode of teleoperation control can be extended to incorporate a large set of intermediate-level, behavior-based, hybrid (qualitative/ quantitative) instructions, such as: move through points A, B, C while avoiding obstacles, pass through door on the left, move at distance $\mathrm{d}$ from wall, follow corridor etc. These commands trigger and make use of respective behavior-based control modes of the robot, incorporating automatic path generation functions. In other words, this mode of teleoperation control is based on some form of basic autonomy (local path planning and reactive sensor-based behaviors etc.) embedded on the slave 


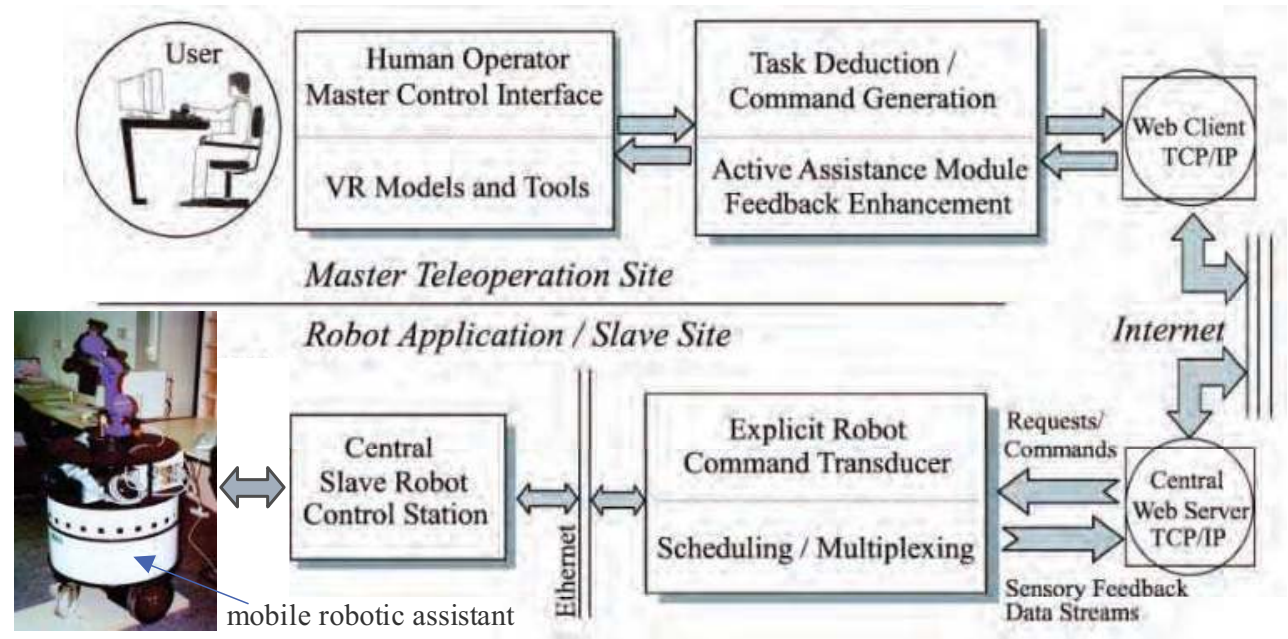

Fig. 11. Schematic representation of the overall teleoperation system for the mobile robotic assistant (adapted from (Tzafestas et al., 2000))

robot. Of course, the master control system should enable this form of remote control, by allowing the human operator to intuitively indicate the robot plan, interpreting his actions and transforming them into appropriate robot instructions that fall into this category. This natural/intuitive human/robot interaction in the context of a shared-autonomy teleoperation control architecture, are issues that will be discussed later on in this chapter.

(d) Semi-autonomous teleoperation, based on a set of high-level qualitative task-based instructions (such as go to location $X$, grasp object A on table B of room C etc.) This set of instructions must be built upon a combination of task-planning, path-generation and environment-perception modules incorporated on the robot control system.

(e) High-level supervisory control, that is, simple monitoring of sensory feedback, and limited human intervention on specific complex situations, requiring difficult decision making and task planning.

Depending on the specific application and the tasks to be performed, a combination of these control modes can be used for on-line monitoring and remote control of the mobile robot. The system, however, also supports a subset of these control modes in an off-line teleprogramming scheme, where the human operator controls the robot task in a simulated environment and checks the validity of his actions before actually sending the commands (registered action plan) to the slave robotic system for real execution. A schematic representation of the overall structure of the teleoperation platform, from the human operator (user) to the mobile robotic assistant, is shown in Fig. 11. To support the "multimodality" of operation, as defined above, the graphical user interface for the teleoperation of the mobile robotic assistant comprises four main components (Fig. 12):

(i) The VR-panel, where the 3D graphical models of the robotic system and its task environment are rendered. This simulation environment constitutes the first modality for inserting instructions (eg. motion commands) to the system in a natural and intuitive way. 
The input devices used were: a joystick for virtual robot motion control, and a Spaceball for virtual camera navigation. Some form of sensory feedback information is also integrated in this virtual environment, like the actual robot position represented by a wireframe graphic model of the robot platform.

(ii) The control-panel, containing a 2D top-view graphical representation of the mobile robot environment (corridors, doors, rooms, obstacles etc.) and a command editing panel. The 2D model contains accurate map information of the whole indoor environment, where the robotic assistant is operating, allowing the user to obtain rapidly a top-view of any required region (using scrollbars or predefined region-buttons). The human operator will also have the ability, if needed, to directly edit commands that must be sent to the robot.

(iii) The sensory-feedback panel, where all the required sensory feedback signals are displayed (for instance a sonar map, indicating the location of obstacles detected by the robot). A visual-feedback panel was also integrated, displaying images obtained by the on-board robot camera. The refresh-rate of this video feedback is of course reduced, since the bandwidth available for communication through the Internet is limited and real-time access to other more critical information, such as actual robot location and sensor status, is indispensable.

(iv) The status panel displaying information on the actual status of the robot in textual form, as well as messages about actions and events.

Fig. 12 shows a snapshot of this prototype human operator interface, developed based on Java technology to facilitate Web-based operation of the system. The 3D graphics rendering routines for the VR panel are implemented using the Java3D API. An enhanced version of this system will constitute in the near future the Internet-based teleoperation control platform for this mobile robotic assistant.

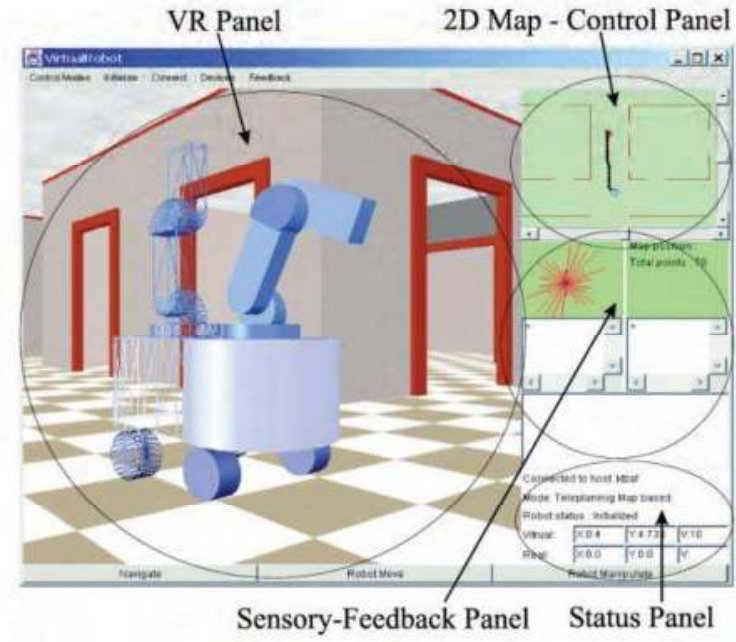

Fig. 12. Multimodal teleoperation interface for the mobile robotic assistant: First prototype implementation (adapted from (Tzafestas, 2001a))

Experiments with this system were very satisfactory, demonstrating convincingly that VR techniques and tools can be used efficiently in the teleoperation of mobile service robots. There 
remain, however, several issues that need further attention and require future research efforts. One of the major difficulties is how to enable both: (a) the human operator to perform actions in a natural and intuitive manner within the master control environment, and (b) the system to interpret these actions, extract critical task-related parameters and synthesize appropriate robot commands. The first issue is related to the design of the human operator interface, where we have opted for the use of VR techniques to enable such an intuitive interaction with the system, while providing active assistance functionalities, as described above. On-line monitoring and analysis of the human operator's actions is then necessary, to deduce a correct robot task plan as specified/indicated by these actions. This means, in other words, to incorporate some form of "intelligence" in the master control environment, capable of performing these task-deduction and robot-command-extraction operations, based on observation of human actions within a simulated virtual representation of the slave site. One approach to this problem of robot action tele-planning from observation and learning of VR-based human demonstration, is described in (Tzafestas, 2001b).

In the same context of "embedding intelligence" in telerobotic interfaces to facilitate the human operator task and enhance system performance, an important issue concerns the development of systems integrating more advanced input/output modalities, inspired from works in the field of virtual reality (for instance, providing active assistance through haptic devices, as simple as a force feedback joystick, or more enhanced like a general-purpose haptic device such as the PHANTOM ${ }^{\circledR}$ devices from SensAble Technologies ${ }^{7}$ ). Such a research effort, involving active haptic display in a mobile robot teleoperation interface, is described in the following section.

\subsubsection{Haptic Teleoperation for Remote Exploration}

As discussed above, the use of VR technologies in mobile robot teleoperation is usually focused on providing mainly visual assistance to the human operator, via 3D modeling and predictive display of the remote environment, either for on-line guidance or for teleprogramming / teleplanning purposes. Since quite recently, this field is evolving towards a more fundamental pursuit of an efficient human-robot cooperation framework, where the system is designed according to schemes aiming to combine in an "optimal" way the capacities of humans and robots. Many researchers are currently concentrating on establishing such a "synergetic and collaborative" telerobotic control framework, as this was done in the telemanipulation case, like for instance the "shared intelligence" scheme proposed in (Bathia et al., 99), describing a VR-human interface for assisting human input in path planning for telerobots. For the teleoperation of a mobile robot, a simple application of this general principle could be to commit the human operator in performing the necessary global planning operations, which are more demanding in terms of complex reasoning and required "intelligence", while other more local tasks such as collision avoidance and trajectory optimisation are dedicated to the telerobotic system.

Our objective in the design of such advanced VR-based teleoperation systems is, always, to devise new methods to support efficient multimodal sensori-motor interaction and provide adequate multi-sensory information to the human operator, which will assist him (a) to more intuitively perceive important features of the remote environment (where the slave robot is moving), and (b) to indicate his intentions and issue teleoperation commands in a direct and natural way.

\footnotetext{
${ }^{7}$ http://www.sensable.com/products/phantom_ghost/phantom.asp
} 


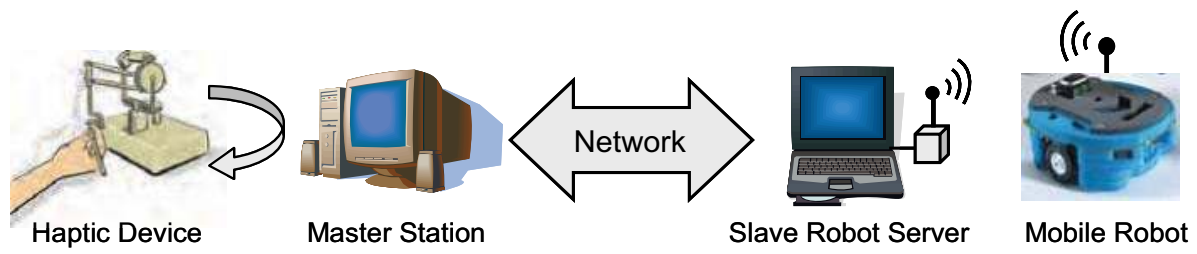

Fig. 13. Overview of a mobile robot "haptic teleoperation" system with master (left) and slave (right) side.

In this context, the application of haptic technologies is now considered as a very promising research direction, offering great potential as a means to revolutionize the way human-robot (and generally human-machine) interfaces are conceived. The term "haptic" comes from the Greek word " $a \varphi \dot{\eta}$ " (touch), and usually refers to the sense of touch, and especially to the human hand that can act on the environment and perceive its physical characteristics. Exploiting human dexterity and manipulative skills within interactive VR systems constitutes a real-challenge for scientists and engineers in the field. Haptics constitutes now a rapidly evolving field of VR technologies, with new research communities active worldwide 8 .

With the spread of low-cost haptic devices, haptic interfaces appear in many areas in the field of robotics. In telerobotics, the integration of haptic technologies and tools has given rise to a new research field that is now often termed as "haptic teleoperation". The main motivation behind this new field is the constant search to enhance transparency and naturalness of teleoperation systems, and promote performance a step further by enriching the human-telerobot interface with new sensori-motor interaction modalities, for efficient human-robot skill transfer and cooperation. Recently, haptic devices have been also used in the field of mobile robot teleoperation, particularly where mobile robots operate in unknown and dangerous environments performing specific tasks. Haptic feedback is shown to improve operator perception of the environment without, however, improving exploration time.

Teleoperated mobile robots are a major tool in the exploration of unknown and risky environments. Mines removal (Smith et al., 1992) and exploration of underwater structures (Lin et al., 97) are two common applications carried out through mobile robots. Robot motion is usually controlled by system operators with the help of a camera mounted on robot or inspecting the area from above. However, although vision systems provide much information of the environment, they require network bandwidth and much attention from the operator. To overcome this problem, haptic devices have been recently introduced as a way of enhancing operators perception of the robot environment. They provide operators with the additional sense of "feeling" the robot workspace, thus making it easier to avoid obstacles and reducing the average number of collisions.

However, the force rendering process yields a problem regarding how the haptic feedback affects the exploration time. Ideally, we would like the presence of force feedback to reduce the exploration time or at least not to increase it. In practice, though, this additional sense often adds more information for operators to interpret and leads to an increase in the navigation time, as for instance in (Palafox et al., 2006). Another important issue in mobile robot teleoperation is the selection of a proper driving mechanism. Usually, operators have to manually drive the mobile robot through obstacles by explicitly specifying the robot

${ }^{8}$ see, for instance: [www.eurohaptics.net] and [www.worldhaptics.org] 
angular and linear velocity. By doing so, they are fully in charge of the robot motion and, as a clear viewpoint of the robot environment may sometimes not be available, they could accidentally drive the robot to collisions or choose longer paths than optimal ones.

In a recent work described in (Mitsou et al., 2006), a teleoperation scheme is presented for the case of remote exploration of a structured polygonal environment by a miniature mobile robot, with the use of a haptic device. During robot exploration, robot sensor measurements are used to build an occupancy grid map of the environment, which is displayed to the operator as a substitute for camera information. The operator can simultaneously exert two different types of commands: an "active" and a "guarded" motion command. Each command receives force feedback independently (without influencing one another) making force origin clear. A behavior-based system is then responsible for controlling the overall motion performed by the slave mobile robot. The commands received from the haptic device act as a general policy that the robot must follow.

An overview of the mobile robot haptic teleoperation system is shown in Fig 13. It consists of two sides: the master side, which contains the haptic device and the master station with the mapbuilding module, and the slave side, which contains the mobile robot and a slave robot server with the behavior and the localization module. A more detailed view of the main system modules and their interconnection is schematically shown in Fig. 14. To develop a teleoperation interface that will facilitate intuitive teleguidance of a mobile robot exploring unknown environments (such as a rectangular maze), the first step was to implement a specific "driving scheme". According to this scheme, the haptic workspace is divided into three types of areas. These areas are not relative to the local coordinate system of the mobile robot, but rather absolute and relative to the local coordinate system of the master workstation monitor. There is a "neutral" area, corresponding to the area in the centre of the haptic workspace and implying a stop command. When selected, the robot immediately stops its motion. When the haptic end-point enters one of the Up, Down, Left or Right areas, the robot changes its orientation and moves according to the operator's command. For instance, if the Up area is selected, the robot will start moving upwards, as watched in the operator monitor. Finally, when the haptic end-point enters one of the "bi-directional" areas, a "combined command" will be issued and sent to the slave side. The robot will be instructed to move towards one direction and simultaneously "wall-follow" a wall. A combined motion command execution is illustrated in Fig. 15 (upper row).

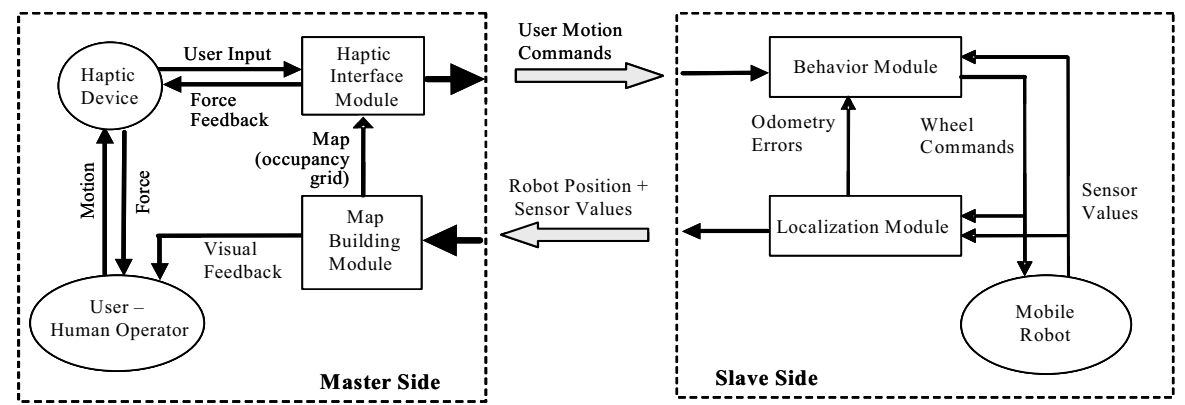

Fig. 14. Mobile Robot Teleoperation System Architecture.

The Map-Building Module is responsible for the creation of an "occupancy grid map" representation of the environment under exploration. It receives robot position and sensors values from the Localization Module of the slave robot server, and updates all affected cells. The map is constructed on-line and is displayed on the Master Computer. Cells that hold 
value greater than a threshold are considered occupied (painted white), cells with value less than this threshold are considered empty (painted black) and cells with no value are considered unvisited (uncertain - painted gray). The constructed map is thus sequentially created and continuously updated on the master control monitor along with the robot position and orientation (Fig. 15). It forms the visual part of the information feedback provided by the interface to assist the human operator in monitoring the task execution and issuing proper navigation commands.

The second principal information feedback channel of the teleoperation interface concerns haptics. As already mentioned, haptic feedback is added to the system to enhance operator's perception of the remote environment, and assist teleoperation of the robot exploration task. The force fed back to the operator is generated based on a 2D virtual joystick model. When the haptic control point exits the "neutral area", a spring force is exerted to the operator attempting to restore end-point position inside this neutral area. The stiffness coefficient of this virtual spring depends on the absence or presence of an obstacle in this direction, that is, on whether the respective motion command is permissible or not. In case that no obstacle hinders the execution of a specific motion command (move forward/backward, turn left/right), the respective spring coefficient is set to a minimum value. This feature enables the operator to feel if he/she is actually exerting an "active" command. In case that an obstacle (e.g. a wall) is detected in the direction of a motion command, the respective spring coefficient is gradually switched to a much larger (max) value. The presence of a wall can thus be viewed as the origin of a virtual repulsive spring force that is applied to the mobile robot. In this case, the operator is feeling a force on his/her hand as if the wall is pushing the robot away, thus conveying the important information that the respective motion command is not permissible.
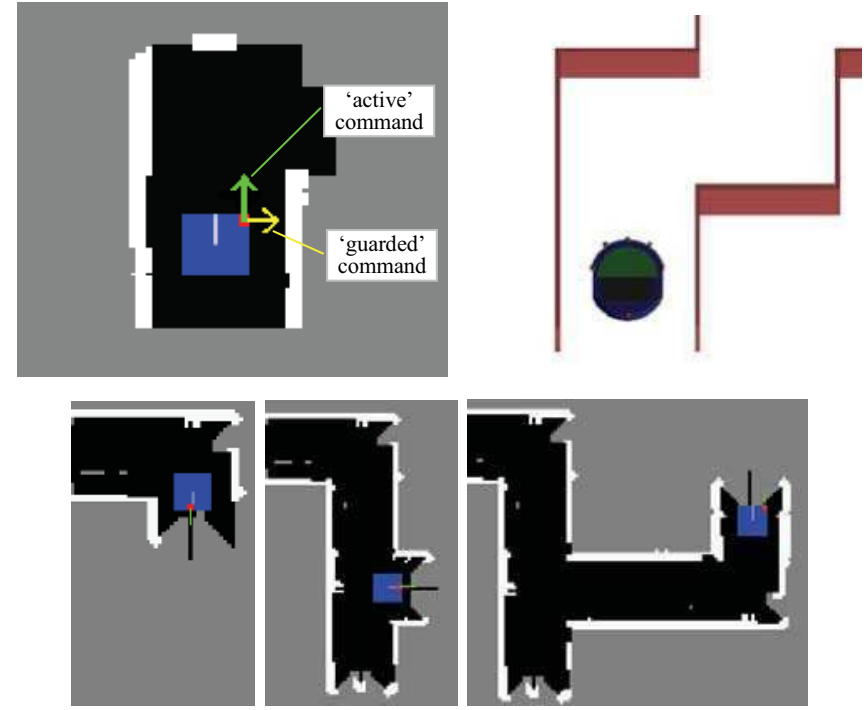

Fig. 15. Example of combined command execution (upper row, in the left, the map on the master station, in the right the VR simulator view), and Sequential Map Creation in the Master Teleoperation Interface (lower row) (adapted from Mitsou et al. 2006). 
It is important to note that, in order to calculate the environmental force that is applied to the human operator via the haptic device, we do not directly use the current sensor values transmitted by the Localization Module. The force is generated according to the values of the cells of the occupancy grid map. If more than $n$ (a threshold we use to deal with noisy sensor values) occupied cells (e.g. forming a virtual wall structure) are found in one direction, then the respective motion command is considered as not permissible. The virtual interaction force is then continuously computed according to the virtual joystick model described above, and sent to the Haptic Device for application. Apart from a spring model, a virtual damper model is also used to smooth the force and avoid large fluctuations in its value.

Additionally, a local robot model is implemented in the master station, to deal with delays in the communication between the master and the slave side. The robot position received from the Localization Module is used to update the local model estimation and the Haptic Interface Module considers the new updated position as the real robot position. In case of great latency in master-slave communication, if no local model is used the operator may not feel a wall coming closer to the robot. However, as the local model estimates the current robot position, the generated force is going to increase and the operator will feel the real distance from the wall.

In the slave side, the Mobile Robot is responsible for the exploration of the unknown environment. The robot used in these experiments was a Hemisson mobile robot ${ }^{9}$. It has a differential drive system, is equipped with six low-range infrared sensors and has no wheel encoders. It sends sensors measurements to a server computer (slave robot server) and receives from it the new speed commands for each wheel. The communication between robot and server computer was performed via a wireless link.

The Behavior Module in the master computer is responsible for the robot motion. Given a policy from the master side, the Behavior Module makes sure that the robot will follow it except for cases of possible collisions. Two different behaviors are implemented: a collision detection behavior and a wall-follow behavior. The first is activated when an obstacle is detected from the three front sensors. In this case, the robot stops when operators' command drives it towards the obstacle but accepts the commands that guide it away from the obstacle. The wall-follow behavior is activated by the operator policy. Under specific policies, the robot can follow walls and automatically turn on corners so as not to lose contact with them.

To test the effectiveness of this approach, two different kinds of experiments were performed. The first one was a comparison between this approach and a common teleoperation method, while the second one constituted a "drive-by-feel" experiment. Both experiments were performed on a simulated mobile robot, with a virtual world (the slave robot environment) on a separate remote computer. Also, a PHANTOM OmniTM haptic device was used for force-feedback generation in the master teleoperation interface. Results show that exploration of unknown environments can be completed in less time when haptic feedback is enabled. The proposed visuo-haptic interface is, thus, found to improve navigation time and decrease operator effort. Comparative evaluation experiments show that the proposed system, with the addition of haptic display and autonomous behaviors in the slave robot system, automates the task of exploration making it much easier for the operators to remotely navigate the robot through an unknown world. Moreover, with no visual information available to the operator, exploration tasks

9 The "Hemisson" webpage: [http:/ / www.hemisson.com] 
consisting of finding the exit in an unknown maze can be completed successfully when force feedback is enabled ('drive by feel' experiment).

Conclusively, the use of VR technologies in such teleoperation interfaces is seen to improve operators perception of the environment, and makes a step towards facilitating and automating teleoperated mobile robot exploration tasks. In the future, work is still needed to extensively test such approaches in practical scenarios, particularly generalizing related system architectures so as to successfully cope with applications involving any type of unstructured environment, as well as to examine system behavior under time delay conditions and develop algorithms to cope with such latency in master-slave round-trip communication.

\section{Conclusion - Future Research Directions}

This chapter has reviewed fundamental concepts and technologies of the general interdisciplinary field described usually by a combination of the terms: Virtual, Augmented or Mixed Reality systems, with the emphasis being on their applications in robot teleoperation. We have first analysed the basics of VR and AR systems, which have shown a great progress of research and development activities during the last decade, demonstrating a constantly increasing repertoire of useful practical applications in diverse domains of human activity. We have then described application scenarios of such VR technologies in the general field of robotics, with the particular focus on telerobotic applications.

We have started by presenting a brief historical survey of the field of telerobotics, and identified the major profits that are related to the integration of VR and AR techniques. Virtual environments can be seen as a means to achieve natural, intuitive, multimodal human/computer (and generally human/machine) interaction; in this sense, a VE can function as an efficient mediator between a human operator and a telerobot, with the main objectives being:

(a) to enhance human perception of the remote task environment and therefore improve transparency of the telerobotic system, by enriching the visual information (complemented by other form of sensory and sensori-motor stimuli) provided to the user, thus conveying complex data in a more natural and easier way;

(b) to contribute to the solution of the time-delay problem in bilateral teleoperation and improve stability of the telerobotic system, by extending the concept of predictive displays and offering a range of control metaphors for both operator assistance and robot autonomy sharing.

We have presented a number of successful case studies, where VR techniques have been effectively applied in telerobotics, for the two main robotic systems categories, namely (i) robot manipulators and (ii) mobile robotic vehicles. A long-distance parallel telemanipulation experiment was described, where an intermediate virtual task representation was used involving direct hand actions by means of a VR glove device. The use of telerobotic technologies in a distance training (virtual and remote laboratory) application has been also demonstrated, with very promising results in this important domain. As related to the field of mobile service robotics, two application scenarios have been described, to highlight the benefits that can result from the integration of VR-based interfaces for the teleoperation of robotic vehicles for a variety of tasks, including service / intervention tasks and remote exploration. The link with the field of haptics is also 
discussed, as an introduction to the new field of haptic teleoperation systems, combining concepts, methods and techniques from the field of haptics as a means to further enhance performance (in terms of a trade-off between transparency and stability), and approach the aforementioned objectives for general telerobotic systems.

In this context, we can assess that the great challenges related to the development of efficient telerobotic systems still remain, as can be described by the long-term vision of creating a framework for an effective cooperation between humans and robots (or telerobots). According to such a visionary objective, an ideal intuitive and natural interaction will enable the system to exploit in a full extent: (a) from one hand, the abilities and skills of the human operator in performing specified operations (in terms of dexterity, as well as rapid decision-making and planning skills in complex environments), and (b) on the other hand, the capacities of automated robotic systems to perform autonomously (with superior speed and accuracy) specific control and manipulation physical tasks. In this direction, VR technologies in conjunction with methods and tools from other related fields (computer vision, artificial intelligence) seem to provide an excellent framework for realising such advances, by: (a) inspiring the development of new human-centered teleoperation (and, generally, human-robot communication) schemes, and (b) supporting the development of advanced user-friendly telerobotic interfaces. VR is now considered as a key technology that will contribute significantly towards such long-term goals, and revolutionize the way human-machine systems are conceived in the years to come.

\section{References}

Anderson, R. J. and Spong, M. W. (1992). “Asymptotic Stability for Force Reflecting Teleoperators", Intern. Journal of Robotics Research, vol. 11, no. 2, pp.135-149, 1992.

Azuma, R. T. (1997). “A Survey of Augmented Reality,” Presence, 6 (4), August 1997.

Bathia, P., and Uchiyama, M. (1999). "A VR-Human Interface for Assisting Human Input in Path Planning for Telerobots", Presence, vol.8, no.3, pp.332-354, June 1999.

Bejczy, A. K., Kim, W. S., and Venema, S. (1990). “The Phantom Robot: Predictive Displays for Teleoperation with Time Delay", 1990 IEEE Int. Conf. on Robotics and Automation, pp. 546-551, 1990.

Burdea, G. and Coiffet, P. (1994). Virtual Reality Technology, John Wiley, 1994.

Ellis, S.R. (1995). "Virtual environments and environmental instruments," Chapter 2, in: Simulated and Virtual Realities: Elements of Perception, K. Carr and R. England (eds.), Taylor \& Francis, London, pp. 11-51, 1995.

Freund, E., and Rossmann, J. (1999). “Projective Virtual Reality: Bridging the Gap between Virtual Reality and Robotics", IEEE Transactions on Robotics and Automation, vol. 15, no. 3, pp. 411-422, June 1999.

Goldberg, K. (2000). "Introduction: The Unique Phenomenon of a Distance", in: The Robot in the Garden. Telerobotics and Telepistemology in the Age of the Internet. K. Goldberg (ed.), MIT Press 2000.

Gracanin, D. and Valavanis, K. P. (1999). “Autonomous Underwater Vehicles”, Guest Editorial, IEEE Robotics and Automation Magazine: Special Issue on Design and Navigation of Autonomous Underwater Vehicles, vol.6, no.2, June 1999.

Hirzinger, G., Brunner, B., Dietrich J., and Heindl, J. (1993). “Sensor-Based Space RoboticsROTEX and Its Telerobotic Features", IEEE Transactions on Robotics and Automation, vol. 9, no. 5, pp. 649-663, 1993. 
Joly, L. D. and Andriot, C. (1995). "Imposing Motion Constraints to a Force Reflecting Telerobot through Real-Time Simulation of a Virtual Mechanism", IEEE Intern. Conf. on Robotics and Automation (ICRA'95)\}, pp.357-362, 1995.

Kheddar, A., Tzafestas, C., Coiffet, P., Kotoku, T., and Tanie, K. (1997). "Multi-Robot Teleoperation Using Direct Human Hand Actions", International Journal of Advanced Robotics, Vol. 11, No. 8, pp. 799-825, 1997.

Kheddar, A., Chellali, R., and Coiffet, P. (2000). "Virtual Reality Assisted Teleoperation", in: VE Handbook, K. M Stanney Edts, December 2000. (See also: http://vehand.engr.ucf.edu/revised2.htm)

Kim, W. S. (1996). "Virtual Reality Calibration and Preview/Predictive Displays for Telerobotics", Presence, vol. 5, no. 2, pp.173-190, 1996.

De Laminat, P. (1993). Automatique - Commande des systèmes linéaires. Edition Hermès, Paris, 1993.

Lin, Q. and Kuo, C. (1997). "Virtual tele-operation of underwater robots," in Proceedings of the 1997 IEEE International Conference on Robotics and Automation (ICRA'97).

Mitsou, N., Velanas, S., and Tzafestas, C.S. (2006). "Visuo-Haptic Interface for Teleoperation of Mobile Robot Exploration Tasks," in Proc: The 15th IEEE International Symposium on Robot and Human Interactive Communication (RO-MAN'06), University of Hertfordshire, Hatfield, United Kingdom, pp. 157-163, 6-8 September 2006.

Niemeyer, G., and Slotine, J. J. (1991). "Stable Adaptive Teleoperation", The IEEE Journal of Oceanic Engineering, vol. 16, no.1, pp.152-162, 1991.

Palafox, O.M., Lee, D., Spong, M. W., Lopez, I., and Abdallah, C. (2006). "Bilateral Teleoperation of Mobile Robots over Delayed Communication Network: Implementation", IROS'2006, Beijing, China, October, 2006. [http://decision.csl.uiuc.edu/ robotics/index.htm]

Rastogi, A., Milgram, P., and Drascic, D. (1996). "Telerobotic Control with Stereoscopic Augmented Reality", SPIE Proceedings, Volume 2653: Stereoscopic Displays and Virtual Reality Systems III, pp 135-146, San Jose, Feb. 1996.

Rosenberg, L. B. (1993). "The use of virtual fixtures to enhance telemanipulation with time delay", ASME Winter Annual Meeting on Haptic Interfaces for Virtual Environments and Teleoperator Systems, New Orleans, Louisiana, 1993.

Sayers, C.P., Lai, A. and Paul, R.P. (1995). "Visual Imagery for Sub-sea Teleprogramming", IEEE International Conf. on Robotics and Automation, May, 1995.

Sayers, C. (1999). Remote control robotics, Springer Verlag, 1999.

Schulz, D., et al. (2000). "Web Interfaces for Mobile Robots in Public Places", IEEE Robotics and Automation Magazine, vol.7, no.1, pp.48-56, March 2000.

Sheridan, T. B. (1992). Telerobotics, Automation and Human Supervisory Control, The MIT Press, 1992.

Simmons, R., et al. (2000). "Lessons Learned from Xavier", IEEE Robotics and Automation Magazine, 7(2), 2000.

Smith, F., Backman, D., and Jacobsen, S. (1992). "Telerobotic manipulator for hazardous environments," Journal of Robotic Systems, 1992

Stein, M. R., (2000). "Interactive Internet Artistry. Painting on the World Wide Web with the PumaPaint Project", IEEE Robotics and Automation Magazine, 7(2), June 2000.

Taylor, K., Dalton, B. (2000). "Internet Robots: A New Robotics Nich", IEEE Robotics and Autom. Magazine, 7(1), (special issue: Robots on the Web), March 2000. 
Thrun, S., et al. (1999). "MINERVA: A Second-Generation Museum Tour-Guide Robot", 1999 IEEE Intern. Conf. on Robotics and Automation (ICRA'99).

Tzafestas, C. S., and Coiffet, P. (1997). "Computing Optimal Forces for Generalized Kinesthetic Feedback on the Human Hand during Virtual Grasping and Manipulation", IEEE Int. Conf. on Robotics and Automation (ICRA'97), pp. 118-123, Albuquerque, New Mexico, 20-25 Avril, 1997.

Tzafestas, C. S., and Valatsos, D. (2000). "VR-based Teleoperation of a Mobile Robotic Assistant: Progress Report," Technical Report DEMO 00/13, National Center for Scientific Research "Demokritos", Inst. of Informatics and Telecommunications, October 2000.

Tzafestas, C. S. (2001a). “Multimodal Teleoperation Interface integrating VR Models for a Mobile Robotic Assistant", in: Proc. 10th International Workshop on Robotics in AlpeAdria-Danube Region (RAAD'2001), Vienna, Austria, May 16-18, 2001.

Tzafestas, C. S. (2001b). "Teleplanning by Human Demonstration for VR-based Teleoperation of a Mobile Robotic Assistant", in: Proc. 10th IEEE International Workshop on Robot-Human Interactive Communication (ROMAN'2001), Bordeaux and Paris, Sept. 18-21, 2001.

Tzafestas, C. S., Palaiologou, N., Alifragis, M. (2006). "Virtual and Remote Robotic Laboratory: Comparative Experimental Evaluation", IEEE Transactions on Education, vol. 49, no. 3, pp. 360- 369, 2006.

Vertut, J., and Coiffet, P. (1984). Les Robots: Téléopération. Tome 3A: Evolution des technologies. Tome 3B: Téléopération assistée par ordinateur. Edition Hermes, Paris, 1984. 


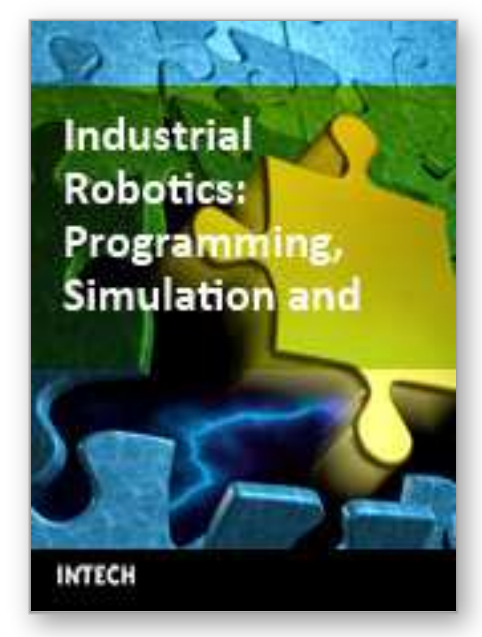

\author{
Industrial Robotics: Programming, Simulation and Applications \\ Edited by Low Kin Huat
}

ISBN 3-86611-286-6

Hard cover, 702 pages

Publisher Pro Literatur Verlag, Germany / ARS, Austria

Published online 01, December, 2006

Published in print edition December, 2006

This book covers a wide range of topics relating to advanced industrial robotics, sensors and automation technologies. Although being highly technical and complex in nature, the papers presented in this book represent some of the latest cutting edge technologies and advancements in industrial robotics technology. This book covers topics such as networking, properties of manipulators, forward and inverse robot arm kinematics, motion path-planning, machine vision and many other practical topics too numerous to list here. The authors and editor of this book wish to inspire people, especially young ones, to get involved with robotic and mechatronic engineering technology and to develop new and exciting practical applications, perhaps using the ideas and concepts presented herein.

\title{
How to reference
}

In order to correctly reference this scholarly work, feel free to copy and paste the following:

Costas S. Tzafestas (2006). Virtual and Mixed Reality in Telerobotics: A Survey, Industrial Robotics: Programming, Simulation and Applications, Low Kin Huat (Ed.), ISBN: 3-86611-286-6, InTech, Available from: http://www.intechopen.com/books/industrial_robotics_programming_simulation_and_applications/virtual_and_ mixed_reality_in_telerobotics_a_survey

\section{INTECH}

open science | open minds

\section{InTech Europe}

University Campus STeP Ri

Slavka Krautzeka 83/A

51000 Rijeka, Croatia

Phone: +385 (51) 770447

Fax: +385 (51) 686166

www.intechopen.com

\section{InTech China}

Unit 405, Office Block, Hotel Equatorial Shanghai

No.65, Yan An Road (West), Shanghai, 200040, China 中国上海市延安西路65号上海国际贵都大饭店办公楼 405 单元

Phone: +86-21-62489820

Fax: +86-21-62489821 
(C) 2006 The Author(s). Licensee IntechOpen. This chapter is distributed under the terms of the Creative Commons Attribution-NonCommercial-ShareAlike-3.0 License, which permits use, distribution and reproduction for non-commercial purposes, provided the original is properly cited and derivative works building on this content are distributed under the same license. 